\title{
Integrated accretion disc angular momentum removal and astrophysical jet acceleration mechanism
}

\author{
P. M. Bellan \\ Applied Physics and Materials Science, Caltech, Pasadena, CA 91125, USA
}

Accepted 2016 March 4. Received 2016 March 4; in original form 2015 March 16

\begin{abstract}
Ions and neutrals in the weakly ionized plasma of an accretion disc are tightly bound because of the high ion-neutral collision frequency. A cluster of a statistically large number of ions and neutrals behaves as a fluid element having the charge of the ions and the mass of the neutrals. This fluid element is effectively a metaparticle having such an extremely small charge-to-mass ratio that its cyclotron frequency can be of the order of the Kepler angular frequency. In this case, metaparticles with a critical charge-to-mass ratio can have zero canonical angular momentum. Zero canonical angular momentum metaparticles experience no centrifugal force and spiral inwards towards the central body. Accumulation of these inward spiralling metaparticles near the central body produces radially and axially outward electric fields. The axially outward electric field drives an out-of-plane poloidal electric current along arched poloidal flux surfaces in the highly ionized volume outside the disc. This out-of-plane current and its associated magnetic field produce forces that drive bidirectional astrophysical jets flowing normal to and away from the disc. The poloidal electric current circuit removes angular momentum from the accreting mass and deposits this removed angular momentum at near infinite radius in the disc plane. The disc region is an electric power source $(\boldsymbol{E} \cdot \boldsymbol{J}<0)$ while the jet region is an electric power sink $(\boldsymbol{E} \cdot \boldsymbol{J}>0)$.
\end{abstract}

Key words: accretion, accretion discs.

\section{INTRODUCTION}

Accretion of mass towards a central object (star or black hole) occurs over a great range of scales, densities, temperatures, magnetic fields, composition, and ionization (Papaloizou \& Lin 1995; Hartmann 2009). This great range suggests that a simple, highly scalable mechanism causes accretion. Accretion is observed to occur in the form of thin axisymmetric discs so the process can be characterized using a cylindrical coordinate system $\{r, \phi, z\}$.

The accretion process must remove angular momentum $m r^{2} \dot{\phi}$ of the accreting material for this material to penetrate to small $r$. This cannot be explained by classical mechanics because $L=m r^{2} \dot{\phi}$ is a conserved quantity in classical mechanics so the azimuthal kinetic energy $L^{2} /\left(2 m r^{2}\right)$ of the accreting material would become infinite as $r \rightarrow 0$. Attempts to explain angular momentum removal by simple viscosity fail because classic viscosity is far too small. Previous models for accretion and angular momentum transport invoke the ideal magnetohydrodynamic (MHD) equations so that the accreting material is assumed to be a perfectly conducting fluid satisfying the ideal MHD Ohm's law

$\boldsymbol{E}+\boldsymbol{U} \times \boldsymbol{B}=0$.

^E-mail: pbellan@caltech.edu
When equation (1) is combined with Faraday's law, the resulting induction equation states that magnetic flux is frozen into the fluid centre of mass.

Steady-state axisymmetric MHD models where an inward accretion flow interacts with a dipole-like poloidal magnetic field fail however because the azimuthal component of equation (1) is $E_{\phi}+U_{z} B_{r}-U_{r} B_{z}=0$. Accretion corresponds to $U_{r}<0$ in the $z=0$ plane, Faraday's law imposes $E_{\phi}=0$, while flow symmetry requires $U_{z}=0$ in the $z=0$ plane so no terms balance the $U_{r} B_{z}$ term associated with an accreting flow (negative $U_{r}$ ). Much effort has gone into developing turbulent (i.e. non-axisymmetric) ideal MHD mechanisms such as the magnetorotational instability (MRI; Hawley, Gammie \& Balbus 1995; Stone et al. 1996; Sano et al. 2000; Balbus 2003) that would transport angular momentum outwards and so remove the angular momentum of accreting material. The MRI was developed primarily in the context of accretion discs surrounding new-born stars; these protoplanetary discs are the progenitors of planets. The MRI was first presented as a linear instability derived analytically in a rotating frame using MHD equations written in this rotating frame while further development was done numerically by typically working in a Cartesian geometry corresponding to a small segment of the rotating frame (shearing box). However, these turbulent mechanisms are complicated and it is not obvious how they would scale over a wide range of parameters. 
While the model presented here is intrinsically different from the MRI, it addresses the same question of how angular momentum can be transported outwards in a protoplanetary disc. The substantial previous work on the MRI has analysed various physical aspects of protoplanetary discs so as to determine relevant approximations of governing equations. We take advantage of some of this previous analysis to justify certain approximations made in the present model. Nominal physical properties have been presented in Balbus $\&$ Terquem (2001). Protoplanetary discs are thin, very weakly ionized, quite cold, have inner radii of a few au, outer radii of 10-100 au and an accretion rate of $10^{-9}-10^{-7} \mathrm{M}_{\odot} \mathrm{yr}^{-1}$. Their magnetic fields are presumed to be of the order of milligauss or higher. For these parameters, the ion-neutral collision frequency $\nu_{\text {in }}$ is much greater than the Kepler frequency $\omega_{\mathrm{K}}=\sqrt{M G / r^{3}}$, where $M$ is the star mass and $r$ is the radial distance from the star; thus, ions are locked to the neutrals which are assumed to be rotating at approximately the Kepler frequency. The ion-neutral collision frequency also greatly exceeds the ion cyclotron frequency $\omega_{\text {ci }}$ so that ions can move across magnetic flux surfaces. However, the electron-neutral collision frequency $v_{\mathrm{en}}$ is much smaller than the electron cyclotron frequency $\omega_{\text {ce }}$ so that electrons are frozen to magnetic flux surfaces. This corresponds to the limit $\omega_{\text {ci }} / \nu_{\text {in }} \ll 1 \ll \omega_{\text {ce }} / \nu_{\text {en }}$ as discussed in section 5 of Wardle (1999). This $\omega_{\text {ce }} / \nu_{\text {en }} \gg 1$ regime is summarized in fig. 2 of Sano \& Stone (2002a) which shows that the Hall term in the generalized Ohm's law greatly exceeds the resistive term for protoplanetary disc parameters. Hall term modifications to the MRI turbulence model have been considered by Balbus \& Terquem (2001), Sano \& Stone (2002a,b), Salmeron \& Wardle (2008), Kunz (2008), Bai (2011, 2014a,b, 2015), Kunz \& Lesur (2013), Lesur, Kunz \& Fromang (2014) and O'Keeffe \& Downes (2014). The Hall term has also been taken into account in a numerical calculation of a circumstellar disc by Tsukamoto et al. (2015) who found that when the Hall term was included, gravitational collapse would occur only when the rotational velocity and magnetic field directions are antiparallel.

Gammie (1996) pointed out that because the source of ionization is outside a protoplanetary disc and because the density of the neutral gas in the disc is very high, the ionization is stratified such that higher ionization exists near the disc surface and dead zones exist around $z=0$.

Observations show that bidirectional jets accompany accretion and the driving force for these jets is presumed to be described by MHD. Swirling of the jets has been proposed as a means for removing disc angular momentum (Blandford \& Payne 1982) but the jets are not observed to have the requisite high angular velocities.

Equation (1) is not a fundamental equation of physics because a substantial number of assumptions must be invoked to derive equation (1). In particular, the derivation of equation (1) involves assuming that Hall currents are negligible. Hall currents may be neglected if the characteristic frequency of the phenomenon under consideration is much less than the ion cyclotron frequency (p. 23 of Freidberg 1987).

The author earlier proposed a non-MHD model (Bellan 2007, 2008a) wherein charged dust grains with a critical charge-to-mass ratio had collisionless trajectories intermediate between Keplerian and cyclotron. This critical charge-to-mass ratio was such that the dust cyclotron frequency was of the order of the Kepler angular frequency. These dust grains would spiral towards the central object with their mechanical angular momentum (i.e. $m r^{2} \dot{\phi}$ ) removed by a magnetic braking mechanism. Direct numerical integration of the equation of motion showed the viability of this mechanism, but exacting requirements on dust density, dust charge-to-mass ratio, and collision mean free path suggested that the mechanism might work only in very specific situations. We present here a substantially revised model that overcomes these shortcomings and should apply to realistic accretion discs composed of dense, weakly ionized gas. Because of its simplicity, the revised model should scale to the great range of parameters associated with accretion. Since readers from the astrophysical disc community may be unfamiliar with certain concepts used in this paper, those concepts not commonly used in astrophysics will be explained in considerable detail. The model will be presented using three equivalent points of view, namely single-particle Lorentz equation, single-particle Hamiltonian, and multifluid. Each of these points of view provides complementary insights and the fact that the model can be presented using all these various points of view demonstrates that the model is robust and not an artefact of any one of these points of view.

The essential point of this paper, an integrated model for accretion and astrophysical jets, results by combining the following concepts:

(i) The fundamental conserved quantity in an axisymmetric system having electric and magnetic fields is not the angular momentum, but instead a different and more generalized quantity called the canonical angular momentum.

(ii) Particles having similar mass exchange momentum via collisions much faster than particles having very different masses so ions in a weakly ionized plasma efficiently share their momentum with neutrals but have negligible momentum exchange with electrons.

(iii) Because ions in a weakly ionized plasma are strongly bound to the neutrals via collisions, the ions and neutrals can be considered as effectively attached to each other.

Concepts (ii) and (iii) show that the plasma is in a Hall regime, a conclusion which is in agreement with Wardle (1999), Balbus \& Terquem (2001), Sano \& Stone (2002a,b), Salmeron \& Wardle (2008), Kunz (2008), Pandey \& Wardle (2008), Konigl (2010), Bai (2011, 2014a,b, 2015), Kunz \& Lesur (2013), Lesur et al. (2014) and O'Keeffe \& Downes (2014) but the analysis of how the plasma behaves in this regime differs completely because of concept (i) which was not taken into account in these previous papers. In contrast to the previous papers which were based on the MRI, a turbulent instability, here the behaviour is not turbulent and instead depends on a dynamo mechanism resulting from basic considerations of the trajectories of charged particles in the combination of gravitational and electromagnetic fields.

This paper is organized as follows: conservation of canonical angular momentum is introduced in Section 2 in the simplest possible context, namely the Lorentz equation with a uniform magnetic field, and it is shown that particles with zero canonical angular momentum in general have inward spiralling trajectories and so behave qualitatively different from particles having the more familiar Kepler or cyclotron orbits. Section 3 shows in a non-rigorous way that the combination of ions and neutrals in a collisional weakly ionized plasma act like a hypothetical collisionless 'metaparticle' having a charge-to-mass ratio orders of magnitude smaller than that of an ion. Section 4 reviews relevant properties of axisymmetric electromagnetic fields and expresses these fields in terms of flux functions. Using the flux functions of Section 4, Section 5 rigorously re-derives the concept of canonical angular momentum conservation introduced in Section 2, applies this concept to the metaparticles, and shows that metaparticles with zero canonical angular momentum have a collisionless inward spiral trajectory. Section 6 considers a two-fluid point of view where one fluid is composed of collisionally bound ions and neutrals while the other fluid consists of neutralizing electrons. Section 6 shows that the Hall term omitted from 
equation (1) becomes critically important as the degree of ionization becomes very small. Section 6 further shows that conservation of canonical angular momentum also pertains to the fluid point of view as do the inward spiral trajectories revealed in the singleparticle equations; the metaparticles of Sections 2 and 5 become the fluid element of Section 6. Section 7 develops fluid equations for torque and angular momentum flux and shows that while the fluid equations conserve global angular momentum, local sources or sinks of angular momentum may exist and these correspond to the magnetic forces discussed in Sections 2, 5 and 6. Section 8 gives an overview of the topology and operation of a laboratory astrophysical jet experiment at Caltech and shows how this topology can be combined with the analysis of the previous sections to construct an integrated, global model for an astrophysical disc-andjet configuration. Section 9 presents order-of-magnitude estimates which show that this model predicts credible quantitative values for the various relevant parameters. Section 10 briefly discusses the charged particle motion responsible for the poloidal magnetic field assumed in the previous sections. Section 11 provides a short summary. Appendix A provides a minor extension to the discussion in Section 2 while Appendix B derives the ion-neutral and electron fluid equations used in Section 6; this derivation results from taking moments of the collisional Vlasov equation.

\section{ANGULAR MOMENTUM, CANONICAL ANGULAR MOMENTUM AND INWARD SPIRAL ORBITS}

Using cylindrical coordinates $\{r, \phi, z\}$, we consider the motion of a particle with mass $m$ and charge $q$ in the plane $z=0$ where (i) at the origin $r=0, z=0$ there is a large mass $M$ providing a gravitational force and (ii) there is also a magnetic field $B=B_{r} \hat{r}+B_{\phi} \hat{\phi}+B_{z} \hat{z}$. This magnetic field is such that in the $z=0$ plane $B_{z}$ is finite and uniform while $B_{r}=B_{\phi}=0$. It must be noted that having $B_{r}=B_{\phi}=0$ in the $z=0$ plane does not exclude the possibility that $B_{r}$ and $B_{\phi}$ are finite outside of the $z=0$ plane. The finite magnetic field component $B_{z}$ in the $z=0$ plane is assumed to be constant and uniform, i.e. in the $z=0$ plane $\partial B_{z} / \partial r=\partial B_{z} / \partial \phi=0$.

The particle equation of motion is

$m \frac{\mathrm{d} \boldsymbol{v}}{\mathrm{d} t}=q(\boldsymbol{E}+\boldsymbol{v} \times \boldsymbol{B})-\frac{m M G}{r^{2}} \hat{r}$.

Because of axisymmetry, there cannot be any electrostatic azimuthal electric field and because $B_{z}$ is assumed steady, there cannot be any inductive azimuthal electric field. Thus, there is no azimuthal electric field, i.e. $E_{\phi}=0$. Because the particle is assumed to stay in the $z=0$ plane, $E_{z}=0$ and $v_{z}=0$. The $r$ and $\phi$ components of equation (2) in the $z=0$ plane are

$m\left(\ddot{r}-r \dot{\phi}^{2}\right)=q E_{r}+q r \dot{\phi} B_{z}-\frac{m M G}{r^{2}}$

$m(2 \dot{r} \dot{\phi}+r \ddot{\phi})=-q \dot{r} B_{z}$

We consider the following two cases: (i) $q=0$ and (ii) $q \neq 0$ by examining the properties of the relevant effective potential. The pertinent effective potential and the results of the direct numerical integration of equation (2) are shown for various subcases in Fig. 1, and the discussion of the effective potentials will cite this figure. Fig. 1 uses normalized coordinates where length is normalized to the initial radius of the particle and time is normalized to the Kepler angular frequency at the starting radius.

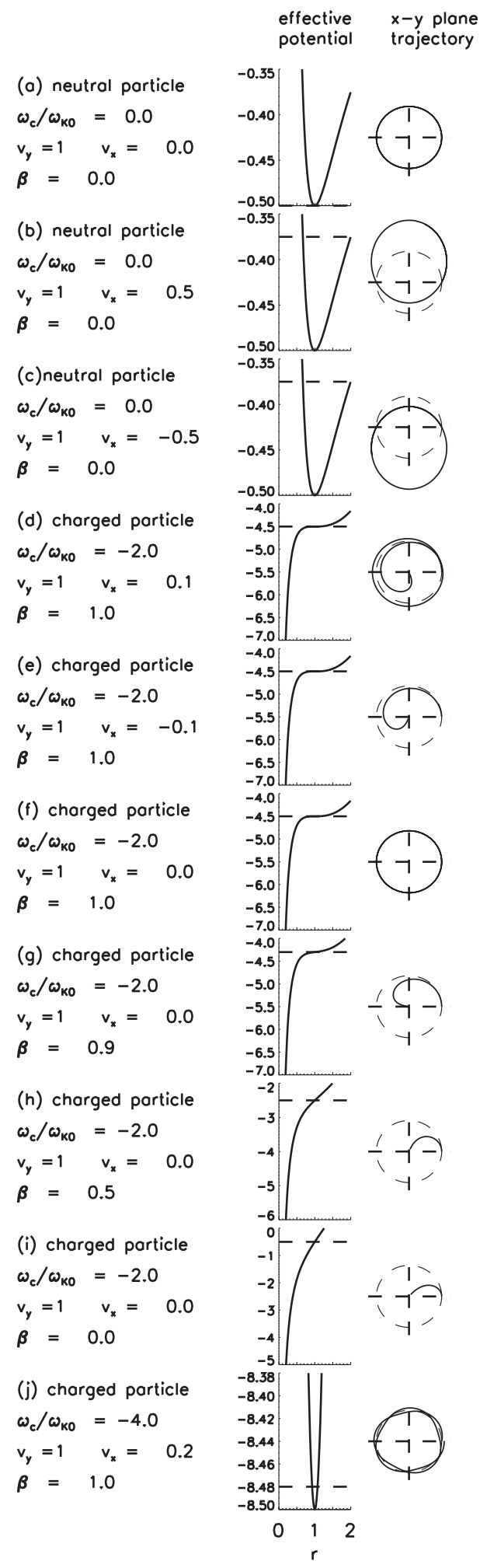

Figure 1. Effective potential $\chi$ and $x-y$ plane particle trajectories for various configurations. The total kinetic plus potential energy is shown as a horizontal dashed line on the effective potential plots. Trajectory is obtained from direct numerical integration of equation (2) with parameters shown to the left of each plot. All trajectories start with $\bar{x}=1, \bar{y}=0$ and $\bar{v}_{y}=\mathrm{d} \bar{y} / \mathrm{d} \tau=1$ but the starting values of $\bar{v}_{x}=\mathrm{d} \bar{x} / \mathrm{d} \tau$ differ and are indicated. The trajectory plots also contain a unit radius reference circle shown by a dashed line. 


\subsection{Case (i): neutral particle}

If the particle is neutral, so $q=0$, then equations (3a) and (3b) reduce to

$\ddot{r}-r \dot{\phi}^{2}=-\frac{M G}{r^{2}}$

$\frac{1}{r} \frac{\mathrm{d}}{\mathrm{d} t}\left(m r^{2} \dot{\phi}\right)=0$.

Equation (4b) gives the classic result that $m r^{2} \dot{\phi}=L=$ const., where $L$ is the angular momentum. Solving for $\dot{\phi}$ gives $\dot{\phi}=L / m r^{2}$ so equation (4a) becomes

$\ddot{r}=\frac{L^{2}}{m^{2} r^{3}}-\frac{M G}{r^{2}}$,

where the term $L^{2} / m^{2} r^{3}$ is the centrifugal force. Equation (5) has a constant $r$ solution (circular orbit) when the centrifugal force and the gravitational force balance each other. Equation (5) can equivalently be expressed as

$\ddot{r}=-\frac{\partial \chi}{\partial r}$,

where $\chi$ is an effective potential given by

$\chi=\frac{L^{2}}{2 m^{2} r^{2}}-\frac{M G}{r}$

and $\chi$ has a minimum at $r=L^{2} /\left(m^{2} M G\right)$. At this minimum, the circular orbits satisfy

$\dot{\phi}^{2}=\frac{M G}{r^{3}}$.

This circular-orbit solution thus has an angular velocity $\dot{\phi}= \pm \omega_{\mathrm{K}}$, where $\omega_{\mathrm{K}}$ is the Kepler angular velocity

$\omega_{\mathrm{K}}=\sqrt{\frac{M G}{r^{3}}}$

These circular Kepler orbits are stable because $\chi(r)$ is at a minimum at $r=L^{2} /\left(m^{2} M G\right)$, i.e. $\partial \chi / \partial r=0$ and $\partial^{2} \chi / \partial r^{2}>0$ at $r=L^{2} /\left(m^{2} M G\right)$. Fig. 1(a) shows the circular Kepler orbit of a neutral particle starting with velocity $v_{y}=\sqrt{M G / r_{0}}$ and $v_{x}=0$; the energy of this particle is located at the bottom of the effective potential and so it cannot bounce in the effective potential well. Fig. 1(b) has in addition finite $v_{x}$ with $v_{x}=0.5 \sqrt{M G / r_{0}}$ while Fig. 1(c) has $v_{x}=-0.5 \sqrt{M G / r_{0}}$ and is seen that as is well known, the orbits become elliptical and can be considered as the particles bouncing back and forth in the effective potential wells because their total energy (kinetic plus potential) exceeds the well minimum.

\subsection{Case (ii): charged particle}

If $q$ is finite, then the right-hand side of equation (3b) must be retained. Let us multiply this equation by the integrating factor $r$ to obtain

$m\left(2 r \dot{r} \dot{\phi}+r^{2} \ddot{\phi}\right)=-q r \dot{r} B_{z}$

which can be expressed as

$\frac{\mathrm{d}}{\mathrm{d} t}\left(m r^{2} \dot{\phi}+\frac{1}{2} q r^{2} B_{z}\right)=0$.

Integration gives

$P_{\phi}=m r^{2} \dot{\phi}+\frac{1}{2} q r^{2} B_{z}=$ const. where $P_{\phi}$ is called the canonical angular momentum and differs from the angular momentum because of the second term $q r^{2} B_{z} / 2$. We now define $\psi$ to be the magnetic flux linked by a circle of radius $r$ so $\psi=B_{z} \pi r^{2}$. The canonical angular momentum can thus also be expressed as

$P_{\phi}=L+\frac{q \psi}{2 \pi}=$ const.

Since $\psi$ depends on position, it is seen that in contrast to case (i), $L$ is no longer a constant of the motion and a charged particle does not make a circular Kepler orbit. Charged particles make more complicated orbits that must be investigated in detail; such an investigation has been presented in Bellan (2007) and in Bellan (2016). We re-arrange equation (3a) as

$\ddot{r}=\frac{q}{m} E_{r}+r \dot{\phi}\left(\dot{\phi}+\omega_{\mathrm{c}}\right)-\frac{M G}{r^{2}}$,

where $\omega_{\mathrm{c}}=q B_{z} / m$ is the cyclotron frequency, solve equation (12) for $\dot{\phi}$ to obtain

$\dot{\phi}=\frac{P_{\phi}}{m r^{2}}-\frac{\omega_{\mathrm{c}}}{2}$,

and substitute for $\dot{\phi}$ in equation (14) to obtain

$\ddot{r}=\frac{q}{m} E_{r}+r\left(\left(\frac{P_{\phi}}{m r^{2}}\right)^{2}-\frac{\omega_{\mathrm{c}}^{2}}{4}\right)-\frac{M G}{r^{2}}$.

A situation of special interest is where $P_{\phi}=0$ in which case equation (15) gives

$\dot{\phi}=-\frac{\omega_{\mathrm{c}}}{2}$

and equation (16) reduces to

$\ddot{r}=\frac{q}{m} E_{r}-\frac{r \omega_{\mathrm{c}}^{2}}{4}-\frac{M G}{r^{2}}$

which has the remarkable feature that there is no centrifugal force as existed in equation (5). The orbital motion will thus be very different from Keplerian. The last two terms in equation (18) both correspond to radially inward forces and so unless $q E_{r} / m$ is particularly strong and outwards, there will be a net inward force and the particle will fall radially inwards to the origin while having an angular velocity given by equation (17).

The electric field $E_{r}$ will be determined by the actual detailed charge distribution. We shall argue that if $E_{r}$ starts from zero, it will build up as inward spiralling particles accumulate near $r=0$ and develop a space charge that repels further inward spiralling particles. Thus, we anticipate that the space charge electric field will build up until it is so large as to make the right-hand side of equation (18) vanish.

It has often been assumed in accretion disc models that the ideal Ohm's law equation (1) applies and that the velocity is the Kepler velocity, i.e. $\boldsymbol{U}=\omega_{\mathrm{K}} r \hat{\phi}$ in which case equation (1) fixes the electric field to be

$\boldsymbol{E}^{\mathrm{Kepler}}=-\boldsymbol{U} \times \boldsymbol{B}=-\sqrt{\frac{M G}{r}} \hat{\phi} \times B_{z} \hat{z}=-\sqrt{\frac{M G}{r}} B_{z} \hat{r}$,

where the superscript Kepler has been used to differentiate this presumption for the electric field from other possibilities. Because equation (19) is such a widely used assumption, we explore the implications of having $E^{\text {Kepler }}$ for the situation where $P_{\phi}=0$. We first allow $P_{\phi}$ to be arbitrary and then afterwards set $P_{\phi}$ to zero. 
Also, to allow for the possibility of an electric field different from $E^{\text {Kepler }}$, we assume the electric field is radial and given by

$E_{r}=-\beta \sqrt{\frac{M G}{r}} B_{z}$,

where $\beta$ is the normalization to the Kepler electric field defined in equation (19) so $\beta=1$ corresponds to having the Kepler electric field while $\beta=0$ would correspond to no electric field.

Let us assume that a particle starts at a radius $r=r_{0}$ with initial velocity $\dot{\phi}=\dot{\phi}_{0}$ and $\dot{r}=\dot{r}_{0}$. Furthermore, we define the Kepler angular frequency at $r_{0}$ to be

$\omega_{\mathrm{K} 0}=\sqrt{\frac{M G}{r_{0}^{3}}}$

and define the normalized radius and normalized time

$\bar{r}=\frac{r}{r_{0}}$

$\tau=\omega_{\mathrm{K} 0} t$

Since $P_{\phi}=$ const., we may evaluate $P_{\phi}$ when the particle starts so $P_{\phi} /\left(m r_{0}^{2}\right)=\dot{\phi}_{0}+\omega_{\mathrm{c}} / 2$. On substituting for $E_{r}$ and $P_{\phi}$ in equation (16) and then dividing by $r_{0} \omega_{\mathrm{K} 0}^{2}$ the normalized equation of motion becomes

$$
\begin{aligned}
\frac{\mathrm{d}^{2} \bar{r}}{\mathrm{~d} \tau^{2}}= & \left(\frac{\dot{\phi}_{0}}{\omega_{K 0} \bar{r}^{3}}-\frac{\beta}{\bar{r}^{1 / 2}}\right) \frac{\omega_{\mathrm{c}}}{\omega_{\mathrm{K} 0}} \\
& +\left(\frac{1}{\bar{r}^{3}}\left(\frac{\dot{\phi}_{0}}{\omega_{\mathrm{K} 0}}\right)^{2}-\frac{1}{\bar{r}^{2}}\right) \\
& +\left(\frac{1}{\bar{r}^{3}}-\bar{r}\right) \frac{\omega_{\mathrm{c}}^{2}}{4 \omega_{\mathrm{K} 0}^{2}} .
\end{aligned}
$$

Let us now choose $\dot{\phi}_{0}=\omega_{\mathrm{K} 0}$ as traditionally assumed for an accretion disc and define the normalized cyclotron frequency $\bar{\omega}_{\mathrm{c}}=$ $\omega_{\mathrm{c}} / \omega_{\mathrm{K} 0}$ so equation (23) reduces to

$$
\frac{\mathrm{d}^{2} \bar{r}}{\mathrm{~d} \tau^{2}}=\left(\frac{1}{\bar{r}^{3}}-\frac{\beta}{\bar{r}^{1 / 2}}\right) \bar{\omega}_{\mathrm{c}}+\frac{1}{\bar{r}^{3}}-\frac{1}{\bar{r}^{2}}+\left(\frac{1}{\bar{r}^{3}}-\bar{r}\right) \frac{\bar{\omega}_{\mathrm{c}}^{2}}{4} .
$$

If the particle is neutral then the terms involving $\bar{\omega}_{\mathrm{c}}$ vanish and the usual Kepler radial equation is recovered. Equation (24) can be expressed in terms of a force associated with an effective potential

$\frac{\mathrm{d}^{2} \bar{r}}{\mathrm{~d} \tau^{2}}=-\frac{\partial \chi}{\partial \bar{r}}$

where the effective potential $\chi$ is

$\chi(\bar{r})=\left(\frac{1}{2 \bar{r}^{2}}+2 \beta \bar{r}^{1 / 2}\right) \bar{\omega}_{\mathrm{c}}+\frac{1}{2 \bar{r}^{2}}-\frac{1}{\bar{r}}+\left(\frac{1}{2 \bar{r}^{2}}+\frac{\bar{r}^{2}}{2}\right) \frac{\bar{\omega}_{\mathrm{c}}^{2}}{4}$.

If $\beta=1$ so that the electric field is a Kepler electric field, all terms on the right-hand side of equation (24) vanish when $\bar{r}=1$, so one might be tempted to conclude that the situation of a particle starting with the initial condition $\bar{r}=1$ and $\dot{\phi}=\omega_{\mathrm{K} 0}$ results in a stable equilibrium, i.e. the particle undergoes circular Kepler orbits forever at $\bar{r}=1$ as was shown in Figs 1(a)-(c) for a neutral particle. However, this is not true if $P_{\phi}=0$, because the equilibrium is not stable when $P_{\phi}=0$.

Using $P_{\phi}=0$ and $\dot{\phi}_{0}=\omega_{\mathrm{K} 0}$, equation (12) gives $\bar{\omega}_{\mathrm{c}}=-2$ so equation (26) reduces to

$\chi(\bar{r})=-4 \beta \bar{r}^{1 / 2}-\frac{1}{\bar{r}}+\frac{\bar{r}^{2}}{2}$.
Taylor expansion of $\chi$ about $\bar{r}=1$ gives

$$
\begin{aligned}
\chi(\bar{r})= & -4 \beta-\frac{1}{2}+(1-\beta)\left[2(\bar{r}-1)-\frac{1}{2}(\bar{r}-1)^{2}\right] \\
& +\frac{1}{4}(4-\beta)(\bar{r}-1)^{3},
\end{aligned}
$$

which depends very sensitively on $\beta$. If $\beta=1$ exactly, then both the first and second derivatives of $\chi$ vanish at $\bar{r}=1$ so $\chi \sim(\bar{r}-1)^{3}$ in which case $\chi$ has no local minimum at $\bar{r}=1$ and so there is no restoring force to push the particle back to $\bar{r}=1$ if it is slightly displaced from $\bar{r}=1$. This situation is fundamentally different from the neutral particle situation seen in Figs 1 (a)-(c) where $\chi$ was at a local minimum at $\bar{r}=1$ and there was a restoring force to push the particle back to $\bar{r}=1$ if it deviated from $\bar{r}=1$.

Specifically, if $\beta=1$ and the particle is at $\bar{r}=1+\delta$, then $\chi$ increases if $\delta>0$ corresponding to a force pushing towards smaller $\bar{r}$, and yet if $\delta<0$, there is still a force pushing the particle towards smaller $\bar{r}$. The plot of equation (27) in Figs 1(d)-(f) shows that $\bar{r}=1$ is just a local flat spot of $\chi$ and that elsewhere $\chi$ is monotonically increasing with $\bar{r}$. If the particle has any finite positive or negative initial $\dot{r}$, it will move away from $\bar{r}=1$. If $\dot{r}>0$, the particle moves to a larger $\bar{r}$ (i.e. moves uphill) until all its kinetic energy is converted into potential energy at which point the particle reflects and then falls all the way down the $\chi(\bar{r})$ potential to $\bar{r}=0$. If the particle has an initial negative $\dot{r}$, it will immediately fall all the way down to $\bar{r}=0$. Thus, if there is the slightest amount of ellipticity to the Kepler orbit (i.e. slightest finite $\dot{r}$ ), a particle with $\bar{\omega}_{\mathrm{c}}=-2$ will spiral all the way down to the origin. Because no real orbit is perfectly circular to all orders, no real orbit would be stable and so a particle with $\bar{\omega}_{\mathrm{c}}=-2$ would always spiral down to the origin. Equivalently, if the plasma temperature differs from being exactly zero or if there are occasional collisions, particles will have some finite $\dot{r}$ and so will fall down to the origin if they have $P_{\phi}=0$.

This instability of the $P_{\phi}=0$ particle in a Kepler electric field is shown in Figs 1(d)-(h). Fig. 1(f) has $\dot{\phi}=\omega_{\mathrm{K} 0}$ and $\dot{r}=0$ with $\beta=1$ and the particle makes a circular orbit. However, if anything is changed slightly, the particle falls to $r=0$. This is because the effective potential is flat, not a minimum, at $\bar{r}=1$ for $P_{\phi}=0$ and $\beta=1$ so it is as if the particle is sitting on a ledge from which it can easily fall off if given the slightest push.

Fig. 1(d) shows an example of a $P_{\phi}=0$ particle having an initial positive radial velocity (here $v_{x}>0$ ) and in an electric field $E^{\text {Kepler }}$ (i.e. $\beta=1$ ) moving to a larger radius, reflecting, and then spiralling all the way down to the origin; the third from bottom figure on the right-hand side of fig. 1 of Bellan (2007) shows the same type of situation. Fig. 1(e) shows an example where a particle in a Kepler electric field has an initial negative radial velocity and immediately falls down the effective potential towards $r=0$. Only if both $\dot{r}=0$ exactly and $\beta=1$ exactly does the particle make a circular orbit.

If $P_{\phi}=0$ and $\beta<1$, then $\chi(\bar{r})$ as given by equation (27) is a monotonically increasing function of $\bar{r}$ so there is always a net radial inward force at all $\bar{r}$; i.e. the flat spot disappears when $\beta<1$. Thus, a $P_{\phi}=0$ particle in a field with $\beta<1$ will fall in towards $r=0$ while maintaining angular velocity $\dot{\phi}=-\omega_{\mathrm{c}} / 2$. At small $\bar{r}$ the $1 / \bar{r}$ term in $\chi(\bar{r})$ as given by equation (27) is dominant which corresponds to the particle simply spiralling in with its radial inward acceleration given by gravity. In summary, if $\beta=1$ and the electric field is Keplerian, a $P_{\phi}=0$ particle will only maintain circular motion if it has exactly zero initial radial velocity; if it has the slightest initial radial velocity either inwards or outwards, the particle will spiral in to $r=0$. If $\beta<1$, a $P_{\phi}=0$ particle will always spiral in. This 
is demonstrated by Figs 1 (g)-(i) which have $\beta=0.9, \beta=0.5$ and $\beta=0$, respectively, with $\dot{r}=0$.

Appendix A shows that it is not only $P_{\phi}=0$ particles that have unstable circular orbits when $\beta=1$. While not of direct relevance to the main theme of this paper, Appendix A shows that particles with $0<P_{\phi} /\left(m r_{0}^{2} \omega_{\mathrm{K} 0}\right) \leq 3 / 4$ also have unstable circular orbits when $\beta=1$. Unlike $P_{\phi}=0$ particles, the finite $P_{\phi}$ particles in the range $0<P_{\phi} /\left(m r_{0}^{2} \omega_{\mathrm{K} 0}\right) \leq 3 / 4$ do not spiral in to the origin when perturbed from their circular orbits but instead bounce back and forth between a finite inner and a finite outer radius.

This inward spiral trajectory of $P_{\phi}=0$ particles occurs because of the cancellation of centrifugal force that would otherwise strongly repel the particle away from $r=0$. Fig. 1(j) shows that when $P_{\phi} \neq 0$ (i.e. $\bar{\omega}_{\mathrm{c}} \neq-2$ ) the particle makes circular orbits that do not change significantly from circular if $\dot{r}$ is finite. Additional demonstration that $P_{\phi} \neq 0$ particles have circular orbits is shown in fig. 1 of Bellan (2007) for a wide range of $\bar{\omega}_{\mathrm{c}}$, where $\bar{\omega}_{\mathrm{c}} \neq-2$.

The analysis in this section was done in the context of a collisionless particle governed by equation (2) and the $P_{\phi}=0$ situation required $\bar{\omega}_{\mathrm{c}}=\omega_{\mathrm{c}} / \omega_{K 0}=-2$. At this point, one might conclude that these results are not relevant to real accretion discs because (i) real accretion discs are very weakly ionized and highly collisional so the collisionless equation (2) would not apply and (ii) electron and ion cyclotron frequencies are typically many orders larger than Kepler frequencies so it would be impossible to have $\omega_{\mathrm{c}} / \omega_{K 0}=-2$. In the next sections of this paper, we show that high collisionality and extremely weak ionization combine in such a way that certain disc fluid elements in a real accretion disc will behave like collisionless particles with $\omega_{\mathrm{c}} / \omega_{K 0}=-2$ so these fluid elements will spiral in towards the origin and thereby create a space-charge radial electric field. Also, because of the extremely weak ionization, equation (1) will be replaced by the resistive Hall Ohm's law so that the electric field no longer has to satisfy equation (1), i.e. no longer has to be given by equation (19). This reduction in the electric field strength will be shown in Section 9 to be equivalent to the situation where the voltage on the terminals of a finite internal resistance battery drops from its open-circuit value when the battery is connected to a load. Section 9 also shows that the balance between blackbody radiation and ohmic dissipation in the internal resistance cause this resistive voltage drop to arrange itself so that $\beta=1 / 2$. The assumption that $B_{\phi}=0$ in the $z=0$ plane does not preclude the possibility that $\partial B_{\phi} / \partial z$ is finite in the $z=0$ plane. It will be assumed that $B_{\phi}$ is an odd function of $z$ implying a radial electric current flows in the $z=0$ plane since $J_{r} \sim-\partial B_{\phi} / \partial z$; this radial current will be constituted by the inward spiralling $P_{\phi}=0$ particles.

\section{COLLISIONAL BINDING OF IONS AND NEUTRALS}

In this section, we will show in a semiquantitative manner how weak ionization and collisions can combine to reduce the effective cyclotron frequency to be of the order of the Kepler frequency; a more precise argument will be given in later sections using Vlasov and fluid theories.

Consider the small volume of weakly ionized highly collisional gas sketched in Fig. 2. This volume has $N_{\mathrm{i}}$ singly charged ions of mass $m_{\mathrm{i}}$ and $N_{\mathrm{n}}$ neutrals of mass $m_{\mathrm{n}}$ and will be called a clump. Suppose an electromagnetic force $\boldsymbol{F}$ imparts a momentum increment $\boldsymbol{F} \Delta t=m_{\mathrm{i}} \Delta \boldsymbol{v}_{\mathrm{i}}$ in a time $\Delta t$ to each ion in the clump so the ions in the clump together gain momentum $N_{\mathrm{i}} m_{\mathrm{i}} \Delta \boldsymbol{v}_{\mathrm{i}}$. Because of the high

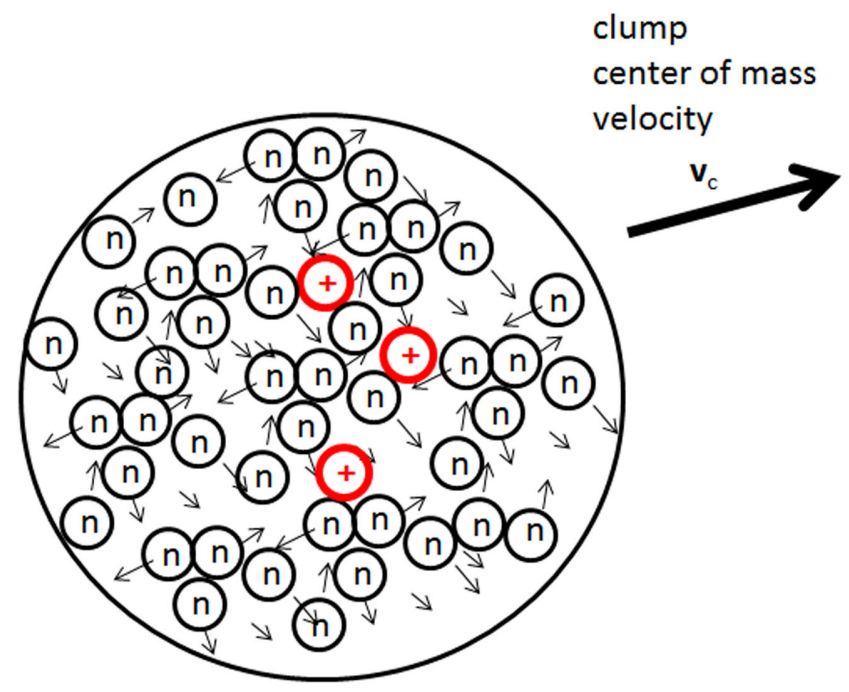

Figure 2. Clump has $N_{\mathrm{n}}$ neutrals and $N_{\mathrm{i}}$ ions with $N_{\mathrm{n}} \gg N_{\mathrm{i}}$. The thermal velocities $v_{\mathrm{T}}$ of the neutrals and ions are shown as small arrows next to the ions and neutrals. These thermal velocities are random relative to the centre-of-mass velocity $\boldsymbol{v}_{\mathrm{c}}$ of the clump and $\left|\boldsymbol{v}_{\mathrm{c}}\right| \gg v_{\mathrm{T}}$.

rate of collisions with neutrals, this momentum gained by the ions is quickly shared with the neutrals in the clump so very quickly

$\left(N_{\mathrm{i}} m_{\mathrm{i}}+N_{\mathrm{n}} m_{\mathrm{i}}\right) \Delta \boldsymbol{v}_{\mathrm{c}}=N_{\mathrm{i}} m_{\mathrm{i}} \Delta \boldsymbol{v}_{\mathrm{i}}$,

where $\boldsymbol{v}_{\mathrm{c}}$ (heavy arrow in Fig. 2) is the mean velocity of all the ions and neutrals in a clump. If a hypothetical metaparticle having mass $\tilde{m}=N_{\mathrm{i}} m_{\mathrm{i}}+N_{\mathrm{n}} m_{\mathrm{n}}$ and charge $\tilde{q}=N_{\mathrm{i}} e$ were in the same electromagnetic field, the momentum increment of the metaparticle would be

$N_{\mathrm{i}} \boldsymbol{F} \Delta t=\left(N_{\mathrm{i}} m_{\mathrm{i}}+N_{\mathrm{n}} m_{\mathrm{n}}\right) \Delta \boldsymbol{v}_{\text {meta }}$.

Comparison of equations (29) and (30) shows that $\Delta \boldsymbol{v}_{\text {meta }}=\Delta \boldsymbol{v}_{\mathrm{c}}$ so the clump responds to the electromagnetic force with a velocity increment as if it were the metaparticle. The electromagnetic force is on the ions, but because the ions are firmly bound to the neutrals via collisions and there are many more neutrals than ions, the effective inertia of the clump is essentially that of the neutrals in the clump.

Metaparticle motion will be determined by

$\frac{\mathrm{d} \boldsymbol{v}_{\mathrm{c}}}{\mathrm{d} t}=\frac{\tilde{q}}{\tilde{m}}\left(\boldsymbol{E}+\boldsymbol{v}_{\mathrm{c}} \times \boldsymbol{B}\right)-\frac{M G}{R^{2}} \hat{R}$,

i.e. the equation of motion of a hypothetical collisionless particle with mass $\tilde{m}$ and charge $\tilde{q}$, where

$\frac{\tilde{q}}{\tilde{m}}=\frac{n_{\mathrm{i}}}{n_{\mathrm{i}} m_{\mathrm{i}}+n_{\mathrm{n}} m_{\mathrm{n}}} e$.

Let us define the ion mass fraction

$\alpha=\frac{n_{\mathrm{i}} m_{\mathrm{i}}}{n_{\mathrm{i}} m_{\mathrm{i}}+n_{\mathrm{n}} m_{\mathrm{n}}}=\frac{n_{\mathrm{i}} m_{\mathrm{i}}}{\rho}$,

where $\rho$ is the mass density. Thus, equation (32) can be written as

$\frac{\tilde{q}}{\tilde{m}}=\alpha \frac{e}{m_{\mathrm{i}}}$

showing that the charge-to-mass ratio of the metaparticle is $\alpha$ times lower than that of a single ion. If $m_{\mathrm{i}}=m_{\mathrm{n}}$, then $\alpha$ is the degree of ionization while if $m_{\mathrm{n}}$ is some small multiple of $m_{\mathrm{i}}$ (e.g. the neutrals are molecules), then $\alpha$ will differ from the degree of ionization by a factor of order unity. 
Assuming weak ionization, and $m_{\mathrm{i}}=m_{\mathrm{n}}$ the condition for the Kepler frequency to be of the order of the clump cyclotron frequency is

$\frac{n_{\mathrm{i}}}{n_{\mathrm{n}}}=\frac{\sqrt{M G / r^{3}}}{e B / m_{\mathrm{i}}}=2 \times 10^{-8} \frac{\sqrt{\frac{M}{\mathrm{M}_{\odot}}}}{\left(\frac{B}{1 \mathrm{mg}}\right)\left(\frac{r}{1 \mathrm{au}}\right)^{3 / 2}}$,

so the clump cyclotron frequency could be of the order of the Kepler frequency for a plausible ionization fraction and plausible magnetic field strength. In this case, it would be wrong to omit the Hall term from Ohm's law because the assumption that the characteristic frequency of the phenomena is small compared to the ion cyclotron frequency would be violated.

Furthermore, the high rate of collisions between ions and neutrals means that ions never manage to complete a cyclotron orbit and so are not individually flux conserving. Since it is the sum of the flux conservation properties of individual particles that provides the flux conservation of the entire plasma, at first sight it would seem that a weakly ionized plasma with highly collisional ions would not conserve magnetic flux. However, being effectively collisionless the metaparticles do complete their very slow cyclotron orbits, and so to the extent that a phenomenon is slower than the metaparticle cyclotron frequency, magnetic flux would be conserved. This is consistent with the conclusions reached by Pandey \& Wardle (2008).

\section{RELEVANT PROPERTIES OF AXISYMMETRIC ELECTROMAGNETIC FIELDS}

Any axisymmetric magnetic field can be decomposed into a poloidal component

$\boldsymbol{B}_{\mathrm{pol}}=\frac{1}{2 \pi} \nabla \psi \times \nabla \phi=\nabla \times\left(\frac{\psi}{2 \pi} \nabla \phi\right)=\nabla \times\left(\frac{\psi}{2 \pi r} \hat{\phi}\right)$

and a toroidal component

$\boldsymbol{B}_{\text {tor }}=\frac{\mu_{0} I}{2 \pi} \nabla \phi$

so

$\boldsymbol{B}=\boldsymbol{B}_{\mathrm{pol}}+\boldsymbol{B}_{\text {tor }}$

Here,

$\nabla \phi=\frac{\hat{\phi}}{r}$

and

$\psi(r, z, t)=\int_{0}^{r} B_{z}\left(r^{\prime}, z, t\right) 2 \pi r^{\prime} \mathrm{d} r^{\prime}$

is the poloidal flux enclosed by a circle of radius $r$ at axial position $z$ while

$I(r, z, t)=\int_{0}^{r} J_{z}\left(r^{\prime}, z, t\right) 2 \pi r^{\prime} \mathrm{d} r^{\prime}$

is the electric current enclosed by a circle of radius $r$ at axial position $z$. The three components of the magnetic field are

$B_{r}=-\frac{1}{2 \pi r} \frac{\partial \psi}{\partial z}$

$B_{\phi}=\frac{\mu_{0} I}{2 \pi r}$

$B_{z}=\frac{1}{2 \pi r} \frac{\partial \psi}{\partial r}$
It is seen from equation (36) that

$r A_{\phi}=\frac{1}{2 \pi} \psi$,

where $A_{\phi}(r, z, t)$ is the azimuthal vector potential. The azimuthal electric field is thus

$E_{\phi}(r, z, t)=-\frac{1}{2 \pi r} \frac{\partial \psi}{\partial t}$.

Equation (43) satisfies the integral form of Ampere's law, namely $2 \pi r B_{\phi}=\mu_{0} I$ while equation (46) satisfies the integral form of Faraday's law, namely $2 \pi r E \phi=-\partial \psi / \partial t$. From Ampere's law, the toroidal current is given as

$J_{\phi}=\mu_{0}^{-1} \hat{\phi} \cdot \nabla \times \boldsymbol{B}_{\mathrm{pol}}=-\frac{r}{2 \pi \mu_{0}} \nabla \cdot\left(\frac{1}{r^{2}} \nabla \psi\right)$

showing that the source of positive $J_{\phi}$ is near where $\psi$ has a maximum and the source of negative $J_{\phi}$ is near where $\psi$ has a minimum. The poloidal current results from the curl of the toroidal magnetic field and is

$J_{\mathrm{pol}}=\frac{1}{2 \pi} \nabla I \times \nabla \phi$

showing that the poloidal current $I(r, z, t)$ acts as the stream-function for $\boldsymbol{J}_{\text {pol }}$ just as the poloidal flux $\psi(r, z, t)$ acts as the stream-function for $\boldsymbol{B}_{\mathrm{pol}}$.

\section{SINGLE-PARTICLE HAMILTONIAN POINT OF VIEW}

The discussion in Section 2.2 presented the essential concept that charged particles with $P_{\phi}=0$ do not have Kepler or cyclotron orbits and instead have a special orbit consisting of a radially inward spiral, but this discussion was restricted to the very simple situation of a uniform magnetic field in the $z$-direction. Section 3 showed that in a weakly ionized plasma where the ions and neutrals are strongly coupled via collisions, the combination of ions and neutrals behaves as a hypothetical metaparticle having the charge-to-mass ratio $\tilde{q} / \tilde{m}$ given by equation (34).

The Lagrangian-Hamiltonian method (Goldstein 1950; Landau \& Lifshits 1960; Schmidt 1979; Bellan 2008b) exploits the consequences of symmetries to provide a deeper insight into particle trajectories than does the equation of motion. The LagrangianHamiltonian method shows that the integrability of the $\phi$ component of the equation of motion is the result of a fundamental principle and not a consequence that a fortuitous integrating factor enables equation ( $3 b)$ to become equation (11).

The Lagrangian for a particle of mass $m$ and charge $q$ in an axisymmetric electromagnetic field and gravitational and electrostatic potentials is (Bellan 2007, 2008a)

$\mathcal{L}=\frac{1}{2} m v^{2}+q \boldsymbol{v} \cdot \boldsymbol{A}(r, z, t)+\frac{m M G}{\sqrt{r^{2}+z^{2}}}+q V(r, z, t)$.

For canonical coordinates $\left\{Q_{\mathrm{i}}\right\}$, Lagrangian theory (Goldstein 1950; Landau \& Lifshits 1960) defines canonical momenta as

$P_{\mathrm{i}}=\frac{\partial \mathcal{L}}{\partial \dot{Q}_{\mathrm{i}}}$

and Lagrange's equations give

$\frac{\mathrm{d} P_{\mathrm{i}}}{\mathrm{d} t}=\frac{\partial \mathcal{L}}{\partial Q_{\mathrm{i}}}$. 
Equation (51) has the important consequence that $\mathrm{d} P_{\mathrm{i}} / \mathrm{d} t=0$ if $\mathcal{L}$ is independent of $Q_{\mathrm{i}}$. Taking $\phi$ to be the canonical coordinate of interest gives

$P_{\phi}=\frac{\partial \mathcal{L}}{\partial \dot{\phi}}$

as the canonical angular momentum. Since the gravitational and electromagnetic fields are independent of $\phi$ (axisymmetry), $\mathcal{L}$ is independent of $\phi$ so $P_{\phi}$ is a constant of the motion. Noting that $\boldsymbol{v} \cdot \boldsymbol{A}$ includes the term $r \dot{\phi} A_{\phi}$, it is seen that

$P_{\phi}=\frac{\partial L}{\partial \dot{\phi}}=m r^{2} \dot{\phi}+q r A_{\phi}=m r^{2} \dot{\phi}+\frac{q}{2 \pi} \psi(r, z, t)=$ const.

This shows that charged particle motion is fundamentally different from neutral particle motion. A neutral particle has $m r^{2} \dot{\phi}=$ const. whereas a charged particle has $m r^{2} \dot{\phi}+q \psi(r, z, t) /(2 \pi)=$ const. The traditional concept that angular momentum $m r^{2} \dot{\phi}$ is conserved is therefore false when particles are charged because it is $m r^{2} \dot{\phi}+$ $q \psi(r, z, t) /(2 \pi)$ that is instead conserved. Furthermore, equation (53) has the meaning that when $q / m \rightarrow \infty$, particles are constrained to stay nearly on a surface of constant $\psi(r, z, t)$ whereas when $q / m \rightarrow 0$, particles have constant $m r^{2} \phi$. The former limit is the basis of particle confinement in fusion devices such as tokamaks while the latter situation is the basis of Kepler orbits. If $P_{\phi}=0$, then the motion is neither particle confinement on a flux surface nor a Kepler orbit.

Equation (53) can be solved for $\dot{\phi}$ to obtain

$\dot{\phi}=\frac{1}{m r^{2}}\left(P_{\phi}-\frac{q}{2 \pi} \psi(r, z, t)\right)$.

The associated Hamiltonian is then

$H=\frac{1}{2} m\left(v_{r}^{2}+v_{z}^{2}\right)+\chi(r, z, t)$,

where the effective potential $\chi(r, z, t)$ is (Bellan 2008a)

$$
\begin{aligned}
\chi(r, z, t)= & \frac{1}{2 m r^{2}}\left[P_{\phi}-\frac{q}{2 \pi} \psi(r, z, t)\right]^{2} \\
& -\frac{m M G}{\sqrt{r^{2}+z^{2}}}+q V(r, z, t) .
\end{aligned}
$$

If the particle is neutral so $q=0$, then $\chi \rightarrow L^{2} /\left(2 m r^{2}\right)-$ $m M G / \sqrt{r^{2}+z^{2}}$, which is the effective potential that results in Kepler orbits. However, if $q \neq 0$, then the particle motion differs from a Kepler orbit.

In Bellan (2007, 2008a), it was noted that particles having $P_{\phi}=0$ have the effective potential

$$
\chi(r, z, t)=\frac{q^{2}}{8 \pi^{2} m r^{2}}[\psi(r, z, t)]^{2}-\frac{m M G}{\sqrt{r^{2}+z^{2}}}+q V(r, z, t) .
$$

For $r \ll a$, where $a$ is the radius of the toroidal current producing the poloidal magnetic field, $B_{z}$ is approximately independent of $r$ so equation (40) gives $\psi(r, z, t) \simeq B_{z}(0, z, t) \pi r^{2}$ in which case

$\chi(r, z, t)=\frac{q^{2}}{8 m}\left[B_{z}(0, z, t)\right]^{2} r^{2}-\frac{m M G}{\sqrt{r^{2}+z^{2}}}+q V(r, z, t)$.

The radial force is given by $F_{\mathrm{r}}=-\partial \chi / \partial r$ and is negative if $-q \partial V / \partial r$ is not too large and positive. Thus, a particle with $P_{\phi}=0$ will spiral in towards $r=0$ if $q E_{r}$ is not too large and positive. The motion is spiral because $P_{\phi}=0$ implies

$m r^{2} \dot{\phi}=-\frac{q}{2 \pi} \psi(r, z, t)$, and using $\psi \simeq B_{z}(0, z, t) \pi r^{2}$ for small $r$ this gives the azimuthal velocity of the inward spiralling particle at small $r$ to be

$$
\dot{\phi} \simeq-\frac{q}{2 m} B_{z}(0, z, t)
$$

as was shown in Section 2.2. Since the motion depends only on the charge-to-mass ratio, one could use equations (49)-(60) to describe the motion of a metaparticle with charge-to-mass ratio $\tilde{q} / \tilde{m}$ as given by equation (32).

Closer examination of equation (57) shows that a particle with $P_{\phi}=0$ will fall down to $r=0, z=0$ no matter what its initial value of $r, z, \dot{r}$ or $\dot{z}$. This is because $\chi$ has a local peak in $r, z$ space at approximately where $\psi$ peaks while $\chi \rightarrow 0$ at infinity and $\chi \rightarrow-\infty$ as $r, z \rightarrow 0$. Particles always make their way to $r=0$, $z=0$ as this is the point of lowest potential energy and there are no potential barriers such as the centrifugal force potential to prevent the particles from falling to $r=0, z=0$.

\section{FLUID EQUATION POINT OF VIEW}

\subsection{Ion-neutral and electron fluid equations and relation to MHD equation of motion and Hall Ohm's law}

Appendix B calculates the respective first moments of the collisional Vlasov equations for ions, neutrals and electrons and gives the sum of the resulting ion and neutral first moments as

$$
\begin{aligned}
\rho\left(\frac{\partial \boldsymbol{U}}{\partial t}+\boldsymbol{U} \cdot \nabla \boldsymbol{U}\right)= & n_{\mathrm{i}} e \boldsymbol{E}+n_{\mathrm{i}} e \boldsymbol{u}_{\mathrm{i}} \times \boldsymbol{B} \\
& +\rho \boldsymbol{g}-\nabla\left(P_{\mathrm{i}}+P_{\mathrm{n}}\right)-n_{\mathrm{e}} e \eta \boldsymbol{J},
\end{aligned}
$$

and the electron first moment as

$0=-n_{\mathrm{e}} e \boldsymbol{E}-n_{\mathrm{e}} e \boldsymbol{u}_{\mathrm{e}} \times \boldsymbol{B}-\nabla P_{\mathrm{e}}+n_{\mathrm{e}} e \eta \boldsymbol{J}$.

Here, $\boldsymbol{u}_{\mathrm{i}}$ is the mean ion velocity, $\boldsymbol{u}_{\mathrm{e}}$ is the mean electron velocity, $\boldsymbol{U}$ is the centre-of-mass velocity, $\rho$ is the mass density, $\boldsymbol{g}$ is the gravitational acceleration, $\eta$ is the electrical resistivity and $P_{\mathrm{e}, \mathrm{i}, \mathrm{n}}$ are pressures defined using random velocities relative to $\boldsymbol{U}$. The electron mass is dropped except in the definition of electron pressure. Assuming quasi-neutrality, the summation of equations (61) and (62) results in the standard MHD equation of motion,

$\rho\left(\frac{\partial \boldsymbol{U}}{\partial t}+\boldsymbol{U} \cdot \nabla \boldsymbol{U}\right)=\boldsymbol{J} \times \boldsymbol{B}-\nabla P+\rho \boldsymbol{g}$,

where $P=P_{\mathrm{i}}+P_{\mathrm{e}}+P_{\mathrm{n}}$. It should be noted that the electric field $\boldsymbol{E}$ cancels when equations (61) and (62) are added so there is no electric field in equation (63); this means that detailed considerations that affect the value of the electric field do not change the form of equation (63). The ion-neutral equation of motion given by equation (61) is what would result if equation (62) were subtracted from equation (63). The summation of equation (61) and (62) additionally causes cancellation of the terms involving $\eta \boldsymbol{J}$; this cancellation results because the momentum lost by electrons on colliding with ions and neutrals is precisely the momentum the ions and neutrals gain from such collisions. Because ions and neutrals collide rapidly with each other $\boldsymbol{u}_{\mathrm{i}} \simeq \boldsymbol{U}$ as discussed in Section 3. Using

$\boldsymbol{J}=n_{\mathrm{e}} e\left(\boldsymbol{U}-\boldsymbol{u}_{\mathrm{e}}\right)$,

it is seen that

$\boldsymbol{u}_{\mathrm{e}}=\boldsymbol{U}-\frac{\boldsymbol{J}}{n_{\mathrm{e}} e}$ 
so equation (62) can be re-arranged as

$\boldsymbol{E}+\boldsymbol{U} \times \boldsymbol{B}-\frac{\boldsymbol{J}}{n_{\mathrm{e}} e} \times \boldsymbol{B}+\frac{1}{n_{\mathrm{e}} e} \nabla P_{\mathrm{e}}=\eta \boldsymbol{J}$,

which is the generalized Ohm's law (e.g. see equations 2-12 on page 21 of Spitzer 1956). The $-\boldsymbol{J} \times \boldsymbol{B}\left(n_{\mathrm{e}} e\right)$ term is called the Hall term. If the Hall, $\nabla P_{\mathrm{e}}$ and $\eta \boldsymbol{J}$ terms are neglected, equation (66) reduces to equation (1) which then gives the result that magnetic flux is frozen into the centre-of-mass frame of the plasma. However, if the Hall term is important, then magnetic flux is no longer frozen into the centre of mass of the plasma. Dropping the Hall term corresponds to assuming that $\boldsymbol{u}_{\mathrm{e}} \simeq \boldsymbol{U}$, i.e. assuming that the difference between electron and ion velocities is much less than the ion velocity. If the ions can move across magnetic field lines whereas the electrons cannot, assuming that the electrons and ions move nearly together is not correct. Equivalently, dropping the Hall term corresponds to assuming that the characteristic frequency of interest is much smaller than the ion cyclotron frequency. This can be seen in an order-ofmagnitude manner by substituting $\boldsymbol{J} \times \boldsymbol{B} \simeq \rho \mathrm{d} \boldsymbol{U} / \mathrm{d} t$ for the Hall term in equation (66) so that this term becomes $\left(-\rho / n_{\mathrm{e}} e\right) \mathrm{d} \boldsymbol{U} / \mathrm{d} t$ and comparing the magnitude of this term to the $\boldsymbol{U} \times \boldsymbol{B}$ term. In a fully ionized plasma, $\rho=n_{\mathrm{i}} m_{\mathrm{i}}$ so the comparison between the two terms is $\left(m_{\mathrm{i}} / e\right) \mathrm{d} \boldsymbol{U} / \mathrm{d} t$ compared to $\boldsymbol{U} \times \boldsymbol{B}$; the ratio of these two terms is $\omega / \omega_{\mathrm{ci}}$, where $\omega_{\mathrm{ci}}=e B / m_{\mathrm{i}}$ is the ion cyclotron frequency and $\omega$ is the characteristic frequency of the phenomenon. However, if the plasma is weakly ionized, then $\rho=n_{\mathrm{i}} m_{\mathrm{i}} / \alpha$, and so the comparison becomes $\left(m_{\mathrm{i}} /(e \alpha)\right) \mathrm{d} \boldsymbol{U} / \mathrm{d} t$ compared to $\boldsymbol{U} \times \boldsymbol{B}$ and now the ratio of the terms becomes $\omega /\left(\alpha \omega_{\mathrm{ci}}\right)$. The effective ion cyclotron frequency is now $\alpha$ times lower than $\omega_{\mathrm{ci}}$ and so if $\alpha$ is sufficiently small, the effective cyclotron frequency can be of the order of the Kepler frequency. Thus, if $\alpha \ll 1$, the Hall term must be retained (as also noted by Pandey \& Wardle 2008) and the plasma centre of mass can move across magnetic flux surfaces.

When the Hall term is important, the behaviour of Ohm's law differs substantively from that of equation (1); it is not just a matter of adding another term and finding a slight modification. For given Kepler velocity $\boldsymbol{U}$ and magnetic field $\boldsymbol{B}$, the structure of equation (1) forces $\boldsymbol{E}$ to have the specific value given by equation (19). This fixing of $\boldsymbol{E}$ by $\boldsymbol{U}$ does not occur when the generalized Ohm's law is used. To see this, it is convenient to express the generalized Ohm's law in the form given by equation (62). Using $\eta=m_{\mathrm{e}} v_{\mathrm{en}} /\left(n_{\mathrm{e}} e^{2}\right)$ and equation (64), it is seen that equation (62) can be re-arranged to be

$v_{\mathrm{en}} \boldsymbol{u}_{\mathrm{e}}+\boldsymbol{u}_{\mathrm{e}} \times \frac{e \boldsymbol{B}}{m_{\mathrm{e}}}=-\frac{e}{m_{\mathrm{e}}} \boldsymbol{E}-\frac{\nabla P_{\mathrm{e}}}{m_{\mathrm{e}} n_{\mathrm{e}}}+v_{\mathrm{en}} \boldsymbol{U}$,

which shows that $\boldsymbol{E}, \boldsymbol{U}$ and $n_{\mathrm{e}}^{-1} \nabla P_{\mathrm{e}}$ can be considered as the independent variables and $\boldsymbol{u}_{\mathrm{e}}$ as the variable to be determined by equation (67). We define

$\kappa=\frac{\nu_{\mathrm{en}}}{e B_{z} / m_{\mathrm{e}}}$

and note that because of symmetry and the assumption that the magnetic field is constant in time, $E_{\phi}=0$ and $\partial P_{\mathrm{e}} / \partial \phi=0$. The $r$ and $\phi$ components of equation (67) are then

$\kappa u_{e r}+u_{e \phi}=-\frac{E_{r}}{B_{z}}-\frac{1}{n_{\mathrm{e}} e B_{z}} \frac{\partial P_{\mathrm{e}}}{\partial r}+\kappa U_{r}$

$\kappa u_{e \phi}-u_{e r}=\kappa U_{\phi}$,

which can be solved for $u_{e r}$ and $u_{e \phi}$ to give

$u_{e r}=-\frac{\kappa}{\kappa^{2}+1}\left(\begin{array}{c}\frac{E_{r}}{B_{z}}-\kappa U_{r}+U_{\phi} \\ +\frac{1}{n_{\mathrm{e}} e B_{z}} \frac{\partial P_{\mathrm{e}}}{\partial r}\end{array}\right)$ $u_{e \phi}=-\frac{1}{\kappa^{2}+1}\left(\begin{array}{c}\frac{E_{r}}{B_{z}}-\kappa U_{r}-\kappa^{2} U_{\phi} \\ +\frac{1}{n_{\mathrm{e}} e B_{z}} \frac{\partial P_{\mathrm{e}}}{\partial r}\end{array}\right)$.

We assume that $|\kappa| \ll 1$ which corresponds to the electrons being able to complete several cyclotron orbits between collisions as discussed in section 5 of Wardle (1999) and as shown in fig. 2 of Sano \& Stone (2002a); this assumption will be further justified in Section 9. We also assume that $U_{\phi}$ and $U_{r}$ are of the order of the Kepler velocity and that the electron pressure gradient can be ignored compared to the other terms. With these assumptions equations (70) become

$u_{e r} \simeq-\kappa\left(\frac{E_{r}}{B_{z}}+U_{\phi}\right)$

$u_{e \phi} \simeq-\left(\frac{E_{r}}{B_{z}}-\kappa U_{r}\right)$.

Equations (71)(a,b) constitute the solution of the Hall Ohm's law and show that unlike equation (1) which fixes $\boldsymbol{E}$ to have the value given in equation (19), here $\boldsymbol{E}$ is an independent variable. If $E_{r} / B_{z}$ is less than or of the order of $U_{\phi}$, then it is seen that $u_{e r} / U_{\phi}$ is of the order of $\kappa$ which corresponds to electrons being frozen to poloidal flux surfaces to the extent that $\kappa \ll 1$. More precise estimates of $u_{e r}$ and $u_{e \phi}$ will be given in Section 9.

\subsection{Ion-neutral fluid equation canonical angular momentum}

Collisions of the locked-together ions and neutrals with electrons has negligible effect on the centre-of-mass motion of the ions and neutrals and this motion is essentially the same as the centre-ofmass motion of the entire plasma. Thus, we may omit the resistivity from equation (61) to obtain

$$
\begin{aligned}
\rho\left(\frac{\partial \boldsymbol{U}}{\partial t}+\boldsymbol{U} \cdot \nabla \boldsymbol{U}\right)= & n_{\mathrm{i}} e \boldsymbol{E}+n_{\mathrm{i}} e \boldsymbol{u}_{\mathrm{i}} \times \boldsymbol{B} \\
& +\rho \boldsymbol{g}-\nabla\left(P_{\mathrm{i}}+P_{\mathrm{n}}\right) .
\end{aligned}
$$

Equation (72) can be written using equation (33) and $\boldsymbol{u}_{\mathrm{i}} \simeq \boldsymbol{U}$ as

$$
\begin{aligned}
\rho\left(\frac{\partial \boldsymbol{U}}{\partial t}+\boldsymbol{U} \cdot \nabla \boldsymbol{U}\right)= & \frac{\rho \alpha}{m_{\mathrm{i}}} e \boldsymbol{E}+\frac{\rho \alpha}{m_{\mathrm{i}}} e \boldsymbol{U} \times \boldsymbol{B} \\
& +\rho \boldsymbol{g}-\nabla\left(P_{\mathrm{i}}+P_{\mathrm{n}}\right) .
\end{aligned}
$$

In a weakly ionized plasma, i.e. a plasma where $n_{\mathrm{i}} \ll n_{\mathrm{n}}, \alpha$ is very small, e.g. in the range $10^{-13}-10^{-8}$ for accretion discs (Pandey \& Wardle 2008). Thus, equation (73) can be considered as a fluid equation for positively charged metaparticles having the charge-tomass ratio of $\alpha e / m_{i}$, i.e. the charge-to-mass ratio given in equation (32). The cyclotron frequency of these metaparticles would thus be $\alpha$ times smaller than the ion cyclotron frequency and so could be of the order of the Kepler frequency.

To demonstrate that canonical angular momentum conservation appears in the context of equation (73), we first note that

$$
\begin{aligned}
& (\boldsymbol{U} \cdot \nabla \boldsymbol{U})_{r}=U_{r} \frac{\partial U_{r}}{\partial r}+\frac{U_{\phi}}{r} \frac{\partial U_{r}}{\partial \phi}+U_{z} \frac{\partial U_{r}}{\partial z}-\frac{U_{\phi}^{2}}{r} \\
& (\boldsymbol{U} \cdot \nabla \boldsymbol{U})_{\phi}=U_{r} \frac{\partial U_{\phi}}{\partial r}+\frac{U_{\phi}}{r} \frac{\partial U_{\phi}}{\partial \phi}+U_{z} \frac{\partial U_{\phi}}{\partial z}+\frac{U_{\phi} U_{r}}{r} \\
& (\boldsymbol{U} \cdot \nabla \boldsymbol{U})_{z}=U_{r} \frac{\partial U_{z}}{\partial r}+\frac{U_{\phi}}{r} \frac{\partial U_{z}}{\partial \phi}+U_{z} \frac{\partial U_{z}}{\partial z} .
\end{aligned}
$$


Consider the $\phi$ component of equation (73), namely

$\rho\left(\begin{array}{c}\frac{\partial U_{\phi}}{\partial t}+U_{r} \frac{\partial U_{\phi}}{\partial r} \\ +U_{z} \frac{\partial U_{\phi}}{\partial z}+\frac{U_{\phi} U_{r}}{r}\end{array}\right)=-\frac{\rho \alpha}{m_{\mathrm{i}}} \frac{e}{2 \pi r}\left(\begin{array}{c}\frac{\partial \psi}{\partial t}+U_{z} \frac{\partial \psi}{\partial z} \\ +U_{r} \frac{\partial \psi}{\partial r}\end{array}\right)$,

where $E_{\phi}$ has been expressed using equation (46), $B_{r}, B_{z}$ have been expressed using equations (42) and (44), and it has been noted that axisymmetry means that $g$ and $\nabla\left(P_{\mathrm{i}}+P_{\mathrm{n}}\right)$ have no $\phi$ components. Since

$\frac{\mathrm{d} \psi}{\partial t}=\frac{\partial \psi}{\partial t}+U_{z} \frac{\partial \psi}{\partial z}+U_{r} \frac{\partial \psi}{\partial r}$,

where

$\frac{\mathrm{d}}{\mathrm{d} t}=\frac{\partial}{\partial t}+U_{z} \frac{\partial}{\partial z}+U_{r} \frac{\partial}{\partial r}=\frac{\partial}{\partial t}+\boldsymbol{U} \cdot \nabla$,

we may write equation (75) as

$\frac{\partial U_{\phi}}{\partial t}+U_{r} \frac{\partial U_{\phi}}{\partial r}+U_{z} \frac{\partial U_{\phi}}{\partial z}+\frac{U_{\phi} U_{r}}{r}=-\frac{\alpha e}{m_{\mathrm{i}}} \frac{1}{2 \pi r} \frac{\mathrm{d} \psi}{\partial t}$.

Note that

$\frac{1}{r} \frac{\mathrm{d}}{\mathrm{d} t}\left(r U_{\phi}\right)=\frac{\partial U_{\phi}}{\partial t}+U_{r} \frac{\partial U_{\phi}}{\partial r}+U_{z} \frac{\partial U_{\phi}}{\partial z}+\frac{U_{\phi} U_{r}}{r}$

so on multiplying by $r$, equation (78) becomes

$\frac{\mathrm{d}}{\mathrm{d} t}\left(r U_{\phi}+\frac{\alpha e \psi}{2 \pi m_{\mathrm{i}}}\right)=0$,

which can be integrated to give

$\frac{P_{\phi}}{m_{\mathrm{i}}}=r U_{\phi}+\frac{\alpha e}{2 \pi m_{\mathrm{i}}} \psi(r, z, t)=$ const.,

which is the same as equation (53). Because equation (80) involves the convective derivative $\mathrm{d} / \mathrm{d} t=\partial / \partial t+\boldsymbol{U} \cdot \nabla$, equation (81) has the meaning that $P_{\phi} / m_{\mathrm{i}}$ is constant as measured by an observer moving with the centre-of-mass velocity $\boldsymbol{U}$, i.e. $P_{\phi} / m_{\mathrm{i}}$ is constant for a fluid element. This confirms the methodology used in Section 2 and shows that the clump of Section 2 is a fluid element, i.e. a volume of the fluid moving with the velocity $\boldsymbol{U}$. Thus, the ionneutral fluid can be considered to be composed of metaparticle fluid elements with charge-to-mass ratio $\alpha$ times smaller than that of an ion. The metaparticle fluid elements execute cyclotron orbits at $\alpha \omega_{\mathrm{ci}}$ with associated guiding centre drifts. It is this metaparticle motion that provides flux conservation for phenomena having characteristic frequencies slower than the metaparticle cyclotron frequency.

The radial component of equation (72) is

$$
\begin{aligned}
\rho\left(\begin{array}{c}
\frac{\partial U_{r}}{\partial t}+U_{r} \frac{\partial U_{r}}{\partial r} \\
+U_{z} \frac{\partial U_{r}}{\partial z}-\frac{U_{\phi}^{2}}{r}
\end{array}\right)= & n_{\mathrm{i}} e E_{r}+n_{\mathrm{i}} e\left(U_{\phi} B_{z}-U_{z} B_{\phi}\right) \\
& +\rho \frac{\partial}{\partial r}\left(\frac{M G}{\sqrt{r^{2}+z^{2}}}\right) \\
& -\frac{\partial}{\partial r}\left(P_{\mathrm{i}}+P_{\mathrm{n}}\right) .
\end{aligned}
$$

For simplicity, let us confine attention to the $z=0$ plane and assume that $U_{z}=0$ from symmetry, pressure is negligible, and $U_{r}$ is small so terms non-linear in $U_{r}$ can be neglected. With these simplifying assumptions, equation (82) reduces to

$\rho \frac{\partial U_{r}}{\partial t}=n_{\mathrm{i}} e E_{r}+n_{\mathrm{i}} e U_{\phi} B_{z}+\rho \frac{U_{\phi}^{2}}{r}-\frac{\rho M G}{r^{2}}$.

We may solve equation (81) for $U_{\phi}$ to obtain

$U_{\phi}=\frac{1}{m_{\mathrm{i}} r}\left(P_{\phi}-\frac{\alpha e \psi(r, z, t)}{2 \pi}\right)$.
If $P_{\phi}=0$, this reduces to $U_{\phi}=-\alpha e \psi(r, z, t) /\left(2 \pi m_{\mathrm{i}} r\right)$, and since $\psi=B_{z} \pi r^{2}$ for small $r$ this gives

$U_{\phi}=-\frac{e B_{z}}{m_{\mathrm{i}}} \frac{\alpha r}{2}$.

Substituting for $U_{\phi}$ in equation (83) gives

$\rho \frac{\partial U_{r}}{\partial t}=n_{\mathrm{i}} e E_{r}-\rho \frac{\left(\alpha \omega_{\mathrm{ci}}\right)^{2} r}{4}-\frac{\rho M G}{r^{2}}$

which is analogous to equation (16) in Section 2.2 with $P_{\phi}=0$, and shows that the centrifugal force $U_{\phi}^{2} / r$ has been cancelled by magnetic forces. The $P_{\phi}=0$ metaparticle consisting of collisionally coupled ions and neutrals has a net radial inward force and so will fall towards $r=0$ while having finite $U_{\phi}$. Since the metaparticle is the same as a fluid element, $P_{\phi}=0$ fluid elements consisting of collisionally coupled ions and neutrals will spiral in towards the central object. Equation (85) shows that these inward spiralling fluid elements (equivalent to previously described metaparticles) have a sense of rotation opposite that of the magnetic field direction; this is consistent with the observation by Tsukamoto et al. (2015) in their numerical calculation that when Hall physics is taken into account, gravitational collapse only occurs when the rotation sense and magnetic field direction are anti-aligned.

The inward spiralling ions and neutrals carry positive charge so as time goes on there will be an accumulation of positive charge near the central object. This accumulation will produce a radial outward electric field $E_{r}$ in the $z=0$ plane that will counteract the gravitational force and so retard the radial inward motion. If there is no mechanism for removing the positive charge, the charge will build up until the outward force from $E_{r}$ balances the gravitational force; this happens when

$E_{r}=\frac{\rho}{n_{\mathrm{i}} e}\left(\frac{\left(\alpha \omega_{\mathrm{ci}}\right)^{2} r}{4}+\frac{M G}{r^{2}}\right)$.

Using $\alpha \omega_{\mathrm{ci}}=-2 \dot{\phi}, \dot{\phi}=\sqrt{M G / r^{3}}$, and $\rho /\left(n_{\mathrm{i}} e\right)=m_{\mathrm{i}} /(\alpha e)$ the open-circuit electric field is given by

$E_{r}=\frac{m_{\mathrm{i}}}{\alpha e}\left(\frac{2 M G}{r^{2}}\right)=-\frac{1}{\dot{\phi}}\left(\frac{M G}{r^{2}}\right) B=E_{r}^{\text {Kepler }}$.

Similarly, a pressure $P_{\mathrm{i}}+P_{\mathrm{n}}$ would build up at small $r$ that would also oppose the inflow. This accumulation of positive charge near $r=0$ could be removed if a conducting path external to the $z=0$ plane existed and provided a conduit for electrons to arrive and neutralize the positive charge. The source for these neutralizing electrons would have to be the electrons left behind when the $P_{\phi}=0$ metaparticles spiralled in towards $r=0$. The out-of-plane conductor would have to originate from the region of left-behind electrons. One can envision an insulated wire that goes from near $r=0$ in the $z=0$ plane, loops up out of the $z=0$ plane, and then back down to the $z=0$ plane at large $r$ where the left-behind electrons are located. The electrons would flow along this wire to neutralize the positive charge at $r=0$. Section 8 will discuss how such a 'wire' can be embodied, but first Section 7 will provide certain useful mathematical relationships that will govern such a 'wire'. When such a wire is in place, the electric field will be partially 'shorted out' in which case the electric field will be reduced from the open-circuit electric field $E_{r}^{\text {open }}$; this reduction will be discussed in Section 9. 


\section{TORQUE AND CANONICAL ANGULAR MOMENTUM}

The relationship between canonical angular momentum, angular momentum, and torque will now be examined. The angular momentum of a particle of species $\sigma$ is

$m_{\sigma} r^{2} \dot{\phi}_{\sigma}=P_{\phi \sigma}-\frac{q_{\sigma}}{2 \pi} \psi(r, z, t)$.

Because $P_{\phi \sigma}=$ const., the time derivative of equation (89) gives for each particle

$$
\begin{aligned}
\frac{\mathrm{d}}{\mathrm{d} t}\left(m_{\sigma} r^{2} \dot{\phi}\right) & =-\frac{q_{\sigma}}{2 \pi}\left(\frac{\partial \psi}{\partial t}+\frac{\partial \psi}{\partial r} v_{r}+\frac{\partial \psi}{\partial z} v_{z}\right) \\
& =-\frac{q_{\sigma}}{2 \pi} \frac{\partial \psi}{\partial t}-q_{\sigma} r\left(B_{z} v_{r}-B_{r} v_{z}\right),
\end{aligned}
$$

where equations (44) and (42) have been used and $\mathrm{d} / \mathrm{d} t=\partial / \partial t+$ $\boldsymbol{v} \cdot \nabla$ is the time derivative as seen by an observer moving with a particle. We multiply equation (90) by $n_{\sigma}$ and sum over species to obtain

$\sum_{\sigma}\left(\frac{\mathrm{d}}{\mathrm{d} t}\left(n_{\sigma} m_{\sigma} r u_{\sigma \phi}\right)-m_{\sigma} r u_{\sigma \phi} \frac{\mathrm{d} n_{\sigma}}{\mathrm{d} t}\right)=-r\left(B_{z} J_{r}-B_{r} J_{z}\right)$.

Note that (i) in summing over the $v_{r}, v_{z}$ of the particles, the random velocity sums to zero so, for example, the sum of all the $v_{r} \hat{r}+v_{z} \hat{z}$ of a species gives the mean velocity $\boldsymbol{u}_{\sigma}$ of that species, (ii) summing $n_{\sigma} q_{\sigma} \boldsymbol{u}_{\sigma}$ gives the current density $\boldsymbol{J}$ and (iii) summing $n_{\sigma} q_{\sigma} \partial \psi / \partial t$ gives zero because of quasi-neutrality.

From equations (39), (42), (44) and (48), it is seen that

$$
\begin{aligned}
-r\left(B_{z} J_{r}-B_{r} J_{z}\right) & =\frac{1}{4 \pi^{2} r}\left(\frac{\partial \psi}{\partial r} \frac{\partial I}{\partial z}-\frac{\partial I}{\partial r} \frac{\partial \psi}{\partial z}\right) \\
& =\frac{1}{4 \pi^{2}} \nabla \cdot(I \nabla \psi \times \nabla \phi) .
\end{aligned}
$$

Using the single species continuity equation equation (B15), equation (91) becomes

$$
\left(\begin{array}{c}
\frac{\partial}{\partial t}\left(\rho r U_{\phi}\right) \\
+\sum_{\sigma} \boldsymbol{u}_{\sigma} \cdot \nabla\left(n_{\sigma} m_{\sigma} r u_{\sigma \phi}\right) \\
+\sum_{\sigma} r m_{\sigma} u_{\sigma \phi} n_{\sigma} \nabla \cdot \mathbf{u}_{\sigma}
\end{array}\right)=\frac{1}{4 \pi^{2}} \nabla \cdot(I \nabla \psi \times \nabla \phi)
$$

or

$$
\begin{aligned}
\frac{\partial}{\partial t}\left(\rho r U_{\phi}\right) & +\sum_{\sigma} \nabla \cdot\left(\boldsymbol{u}_{\sigma} r m_{\sigma} u_{\sigma \phi} n_{\sigma}\right) \\
& =\frac{1}{4 \pi^{2}} \nabla \cdot(I \nabla \psi \times \nabla \phi) .
\end{aligned}
$$

If we ignore electron mass and invoke the assumption that collisions cause the ions and neutrals to have the same velocity $\boldsymbol{U}$, then

$\sum_{\sigma}\left(\boldsymbol{u}_{\sigma} r m_{\sigma} u_{\sigma \phi} n_{\sigma}\right)=\boldsymbol{U} U_{\phi} r \sum_{\sigma} m_{\sigma} n_{\sigma}=\rho r U_{\phi} \boldsymbol{U}$

so equation (94) becomes

$\frac{\partial}{\partial t}\left(\rho r U_{\phi}\right)+\nabla \cdot\left(\rho r U_{\phi} \boldsymbol{U}\right)=\frac{1}{4 \pi^{2}} \nabla \cdot(I \nabla \psi \times \nabla \phi)$.

The quantity $\rho r U_{\phi}$ is the angular momentum density and $\rho r U_{\phi} \boldsymbol{U}$ is the angular momentum flux. If the right-hand side of equation (96) were zero, this equation would be a conservation equation for angular momentum density. The fact that the right-hand side of this equation can be finite implies that angular momentum is not necessarily conserved at any given location.
We next show that equation (96) is consistent with equation (63), the MHD equation of motion. Using equation (74b) the $\phi$ component of equation (63) is

$\rho\left(\frac{\partial U_{\phi}}{\partial t}+U_{r} \frac{\partial U_{\phi}}{\partial r}+U_{z} \frac{\partial U_{\phi}}{\partial z}+\frac{U_{\phi} U_{r}}{r}\right)=J_{z} B_{r}-J_{r} B_{z}$.

Multiplying by $r$, this becomes

$\rho\left(\frac{\partial}{\partial t}+U_{r} \frac{\partial}{\partial r}+U_{z} \frac{\partial}{\partial z}\right)\left(r U_{\phi}\right)=r\left(J_{z} B_{r}-J_{r} B_{z}\right)$.

Adding $r U_{\phi}$ times equation (B16), the MHD continuity equation, and using equation (92) gives

$\left(\begin{array}{c}\frac{\partial}{\partial t} \\ +\boldsymbol{U}^{\prime} \cdot \nabla\end{array}\right)\left(\rho r U_{\phi}\right)+\rho r U_{\phi} \nabla \cdot \boldsymbol{U}=\frac{1}{4 \pi^{2}} \nabla \cdot(I \nabla \psi \times \nabla \phi)$

and re-arranging gives equation (96) thereby establishing that equation (89) is consistent with the MHD equation of motion. Integrating equation (96) over the entire volume for which $I$ is finite, so $I$ is zero on the surface of this volume, shows that

$\int \rho r U_{\phi} \mathrm{d}^{3} r=$ const.

The right-hand side of equation (96) is a torque because it produces a change in angular momentum. The fact that this torque can be expressed as a divergence means that the torque associated with the magnetic force conserves angular momentum over the entire volume for which $I$ is finite but not locally, because locally this term can act as a source or sink for angular momentum. Thus, equation (96) shows that angular momentum is not locally conserved while equation (100) shows that angular momentum is globally conserved. In other words, local torques can exist but the sum of all these local torques over the entire volume for which $I$ is finite must vanish.

\section{RELATION TO CALTECH ASTROPHYSICAL JET EXPERIMENT AND TO MHD JET SIMULATIONS}

We now construct an integrated model for accretion and jets by combining the results of Sections 2-7 with insights obtained from a Caltech astrophysical jet laboratory experiment (Hsu \& Bellan 2002; You, Yun \& Bellan 2005; Kumar \& Bellan 2009). Fig. 3 sketches the laboratory experiment showing that it consists of the following:

(i) a copper disc in the $z=0$ plane,

(ii) a coplanar copper annulus surrounding the disc and separated by a small gap,

(iii) a below-disc coil (not shown) with a toroidal current that produces a poloidal field linking the disc and the annulus (a typical poloidal flux surface is indicated),

(iv) eight holes in the disc and eight holes in the annulus; these holes supply gas via fast transient valves (the holes are not shown in Fig. 3 for reasons of clarity),

(v) a power supply that applies a voltage across the gap to drive a poloidal electrical current shown by the arrows in Fig. 3.

The sequence of operation is as follows: first, the poloidal field linking the disc to the annulus is established by having a toroidal electric current flow in the coil beneath the disc. This is done on a relatively slow time-scale $(5 \mathrm{~ms})$ so the poloidal magnetic field produced by the coil can soak into the copper disc and copper 


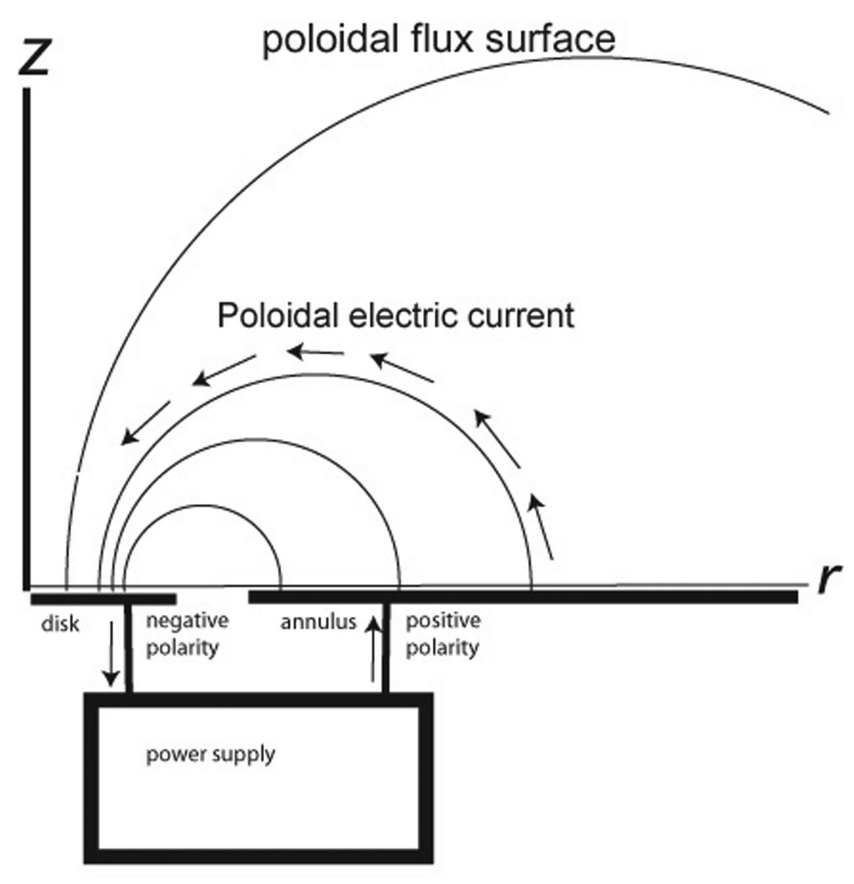

Figure 3. Layout of Caltech astrophysical jet experiment. A coil (not shown) located below the gap between disc and annulus has a toroidal current that creates the poloidal magnetic field shown by semicircles in the positive $z$ region. Gas is injected through holes (not shown) in the disc and the annulus.

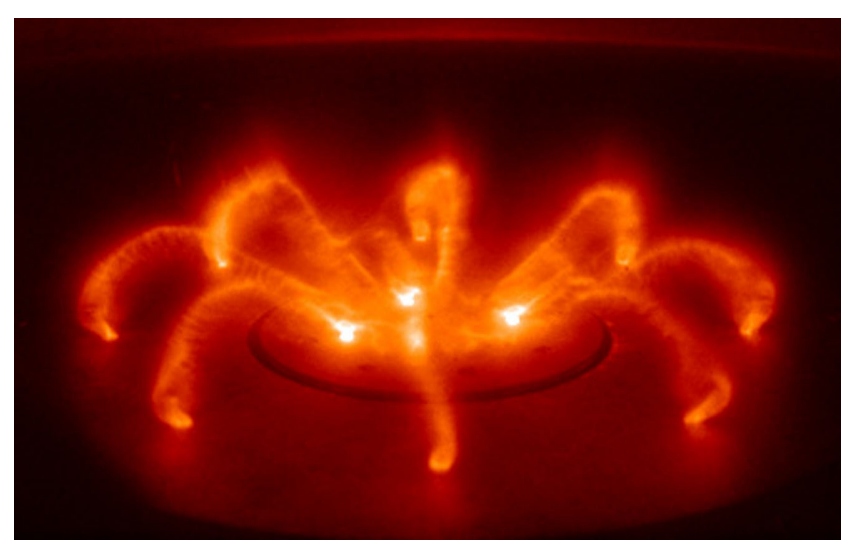

Figure 4. Eight arched magnetic flux tubes trace out shape of initial poloidal field sketched in Fig. 3. The spider legs go from eight gas holes in the disc to eight gas holes in the annulus. This stage is very transient.

annulus. The fast gas valves then inject on an approximately $1 \mathrm{~ms}$ time-scale a small amount of neutral gas from the eight holes in the disc and the eight holes in the annulus. High voltage is then applied across the gap between the disc and the annulus to break down the neutral gas and form an initial plasma consisting of eight arched, plasma-filled flux tubes linking the eight holes in the disc to eight corresponding holes in the annulus as shown by the photo in Fig. 4. These arched, plasma-filled flux tubes are called 'spider legs' because of their shape.

After the spider legs have formed, the configuration evolves as shown in Fig. 5. The inner parts of the spider legs attract each other (4.5-6.5 $\mu$ s frames) because they have parallel electric currents. At the same time, the major radius of the spider legs increases because of the pressure of the toroidal field associated with the poloidal

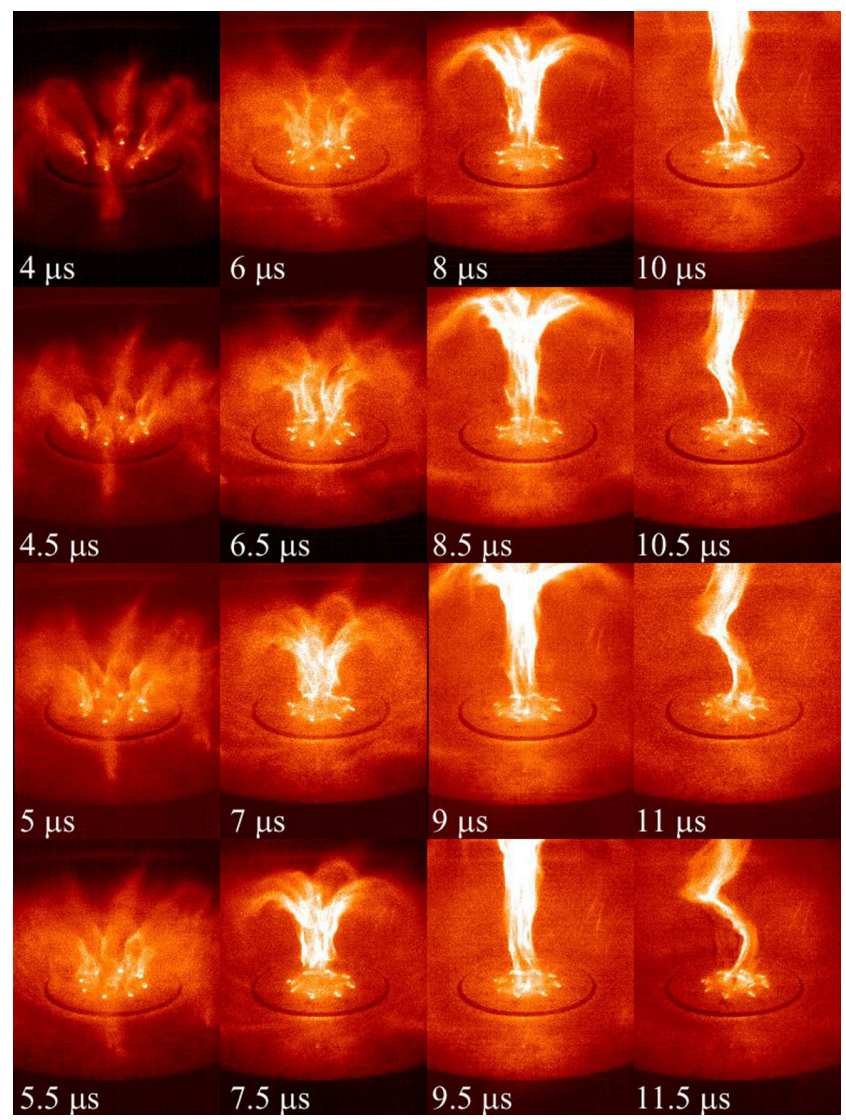

Figure 5. Time development of jet (figure from You et al. 2005).

current flowing along the spider legs (or equivalently because of the $J_{r} B_{\phi}$ force); this is essentially the 'magnetic tower' mechanism of Lynden-Bell (2003) where the toroidal field pressure inflates poloidal flux surfaces since $J_{r} B_{\phi}=-\left(2 \mu_{0}\right)^{-1} \partial / \partial z\left(B_{\phi}^{2}\right)$ provides an axial force pointing from regions of large $B_{\phi}^{2}$ to regions of small $B_{\phi}^{2}$. The inner spider legs merge to form a stalk which lengthens as a result of the gradient in toroidal field. This lengthening stalk constitutes the jet as shown in Fig. $5(6.5-9.5 \mu \mathrm{s})$. At late times the jet undergoes a kink instability $(10-10.5 \mu \mathrm{s})$; this kinking and its consequences have been discussed in Hsu \& Bellan (2002) and in Moser \& Bellan (2012) and will not be discussed here.

The jet behaviour shown in Fig. 5 has been studied in detail and found to be in good agreement with the predictions of ideal MHD. In particular, a high-resolution ideal MHD numerical code originally designed to model astrophysical jets provides a good quantitative description of the Caltech jet experiment including jet radius, position, velocity, acceleration, collimation, pressure, density, magnetic field and current (Zhai et al. 2014). However, the ideal MHD equations were not capable of modelling the $z<0$ 'engine' region consisting of electrodes, power supply, gas injection and coil. A localized artificial source term for the curl of equation (1) (induction equation) had to be introduced in what was effectively the $z<0$ region to represent the engine region. We argue here that this inability of equation (1) to model the $z<0$ laboratory hardware is not because the laboratory hardware fails to correspond to a real astrophysical jet but rather because equation (1) is fundamentally incapable of modelling the engine region of the jet.

We claim that real bidirectional astrophysical jets and their associated accretion disc can be considered equivalent to the Caltech 


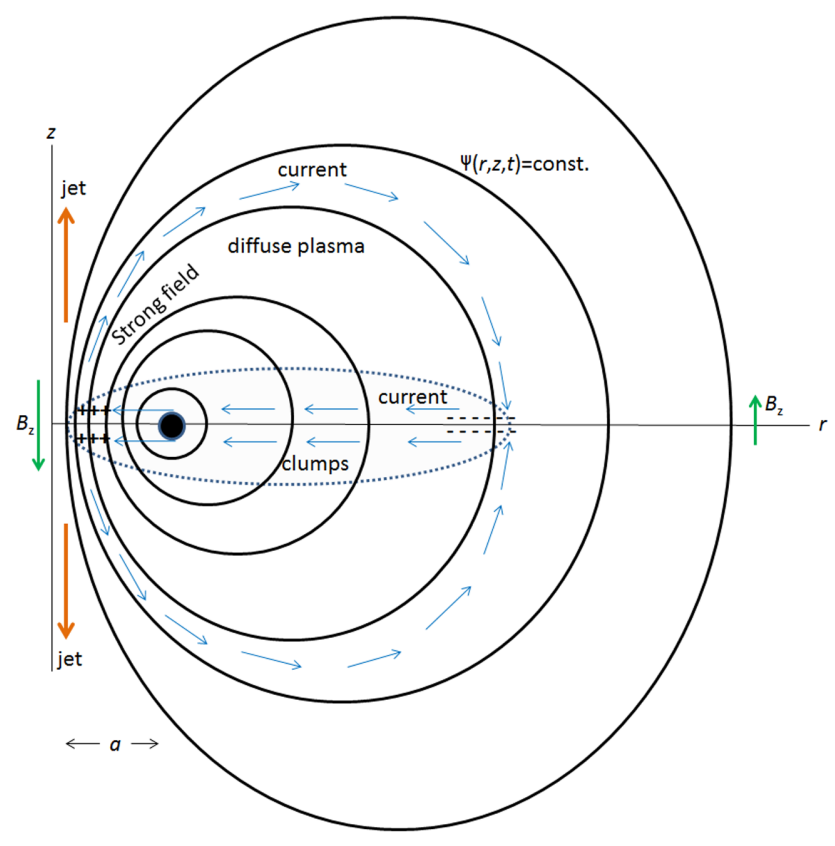

Figure 6. Dense weakly ionized gas inside dotted line ellipse. Positively charged clumps with zero canonical angular momentum spiral in towards the origin and accumulate there providing a net positive charge that drives currents away from the $z$-axis. These currents drive astrophysical jets away from the $z$-axis and flow back to large radius along poloidal flux surfaces. The inward current $J_{r}$ is carried by diffuse plasma in regions $r \gg a$. On the $z=0$ plane the magnetic torque $-J_{r} B_{z}$ removes positive angular momentum where $J_{r} B_{z}>0$ and deposits positive angular momentum where $J_{r} B_{z}<0$. The radial current on the $z=0$ plane is always the same sign, but $B_{z}$ reverses direction on passing $r=a$.

jet if the Caltech jet setup sketched in Fig. 3 is reflected about the $z=0$ plane in such a way that $I(r,-z, t)=-I(r, z, t)$ and $\psi(r$, $-z, t)=\psi(r, z, t)$ and, in addition, the Hall term is retained in a thin layer at the $z=0$ plane but not in the volume external to this thin layer. Accreting matter plays the role of the injected gas in the Caltech experiment while the gravitationally powered inward fall of positive, collisionally coupled ion/neutral fluid elements (clumps with $P_{\phi}=0$ ) plays the role of the experiment power supply in Fig. 3. The poloidal current directions are reversed from the Caltech experiment; this polarity reversal does not affect the dynamics because magnetic forces scale as the square of the currents, e.g. $J_{r} B_{\phi}=-\left(2 \mu_{0}\right)^{-1} \partial / \partial z\left(B_{\phi}^{2}\right)$ where $B_{\phi}$ is given by equation (43).

This copy of the Caltech jet configuration and its suitably reflected mirror is shown in Fig. 6. Here, $B_{\phi}, B_{r}$ and $J_{z}$ are antisymmetric with respect to $z$, while $B_{z}, J_{r}$ and $J_{\phi}$ are symmetric with respect to $z$ in accordance with $\psi(r, z, t)$ being symmetric with respect to $z$ and $I(r, z, t)$ being antisymmetric with respect to $z$. These symmetries both drive bidirectional astrophysical jets and conform to the field orientations of the Caltech plasma jet experiment. The experimental volume of the lab experiment corresponds to the $|z|>0$ region of Fig. 6 while the external hardware and circuitry of the experiment (gas valves, disc, annulus, power supply, coil) corresponds to a thin planar region at $z=0$. The leftward horizontal current at $z=0$ in Fig. 6 corresponds to the current flow in the cables from the power supply in the Caltech experiment (see Fig. 3). Thus, the Caltech experiment power supply is replaced by a dynamo that drives current in the $z=0$ plane. The gas source in the Caltech experiment (inner set of eight holes) is replaced by inward accreting material.
The current in Fig. 6 can be modelled as

$\mu_{0} I=\lambda \psi(r, z, t) \tanh \left(\frac{z}{w}\right)$,

where $\lambda$ is a constant and $w$ is the width of the thin layer at $z=0$. This prescription provides the assumed $z$-antisymmetry of $I$ while concentrating the change in $I$ polarity to the thin layer centred about the $z=0$ plane. Surfaces of constant $I(r, z, t)$ are parallel to surfaces of constant $\psi(r, z, t)$ for $|z| \gg w$, but orthogonal for $|z|<w$. This choice of symmetries produces radial magnetic forces $\sim J_{\phi} B_{z}-J_{z} B_{\phi}$ symmetric with respect to $z$ and axial magnetic forces $J_{r} B_{\phi}-J_{\phi} B_{r}$ antisymmetric with respect to $z$ (recall that $J_{r}$, $J_{\phi}$ and $B_{z}$ are symmetric with respect to $z$, while $J_{z}, B_{r}$ and $B_{\phi}$ are antisymmetric with respect to $z$ ). Near the $z$-axis, the radial magnetic forces are inwards towards the $z=0$ axis (i.e. pinching) while the axial magnetic forces point away from the $z=0$ plane (i.e. point in the direction to drive the bidirectional jets). As discussed in Kumar \& Bellan (2009) and in Zhai et al. (2014), radial pinching produces higher on-axis pressures near $z=0$ than away from $z=0$. The onaxis pressure is an even function of $z$ and is maximum at small $|z|$. The force resulting from this antisymmetric axial pressure gradient accelerates the bidirectional jets away from the $z=0$ plane. This on-axis pressure gradient force is of the same order as and works in tandem with the off-axis magnetic axial force (see pp. 334-338 of Bellan 2008b). The on-axis pressure gradient force is required because the axial magnetic force $J_{r} B_{\phi}-J_{\phi} B_{r}$ vanishes on the $z$-axis as all of $J_{r}, B_{\phi}, J_{\phi}$ and $B_{r}$ are zero on the $z$-axis.

We choose a $\psi$ polarity so that $B_{z}$ is negative at small $r$ and positive at large $r$ as shown in Fig. 6. This choice means that $\psi$ is negative everywhere and has an absolute minimum at the radial location where $B_{z}$ reverses sign, i.e. at the black circle in Fig. 6 . This radius at which $\psi$ is an absolute minimum will be denoted $a$. Because $\psi$ is negative everywhere, equation (59) shows that $P_{\phi}=0$ clumps have positive angular momentum $\tilde{m} r^{2} \dot{\phi}$ everywhere.

In the $z=0$ plane, $B_{r} J_{z}=0$ and $U_{z}=0$ since $B_{r}, J_{z}$ and $U_{z}$ are antisymmetric with respect to $z$. At radii larger than the jet radius there are no sources or sinks of fluid in the $U_{z}$ plane so $\partial U_{z} / \partial z=0$ in the $U_{z}$ plane at radii larger than the jet radius (the jet radius is close to $r=0$ and much smaller than $a$ ).

Since $\partial \psi / \partial z=0$ in the $z=0$ plane, equation (101) gives

$$
\left(\frac{\partial I}{\partial z}\right)_{z=0}=\frac{\lambda}{\mu_{0} w} \psi(r, 0, t)
$$

and so in the $z=0$ plane and away from the bidirectional jets (which is a region where $\partial U_{z} / \partial z \neq 0$ ), equation (96) becomes

$$
\frac{\partial}{\partial t}\left(\rho r U_{\phi}\right)+\frac{1}{r} \frac{\partial}{\partial r}\left(\rho r^{2} U_{r} U_{\phi}\right)=\frac{1}{8 \pi^{2} r} \frac{\lambda}{\mu_{0} w} \frac{\partial \psi^{2}}{\partial r} .
$$

Setting $\lambda$ to be negative gives negative $\partial \psi^{2} / \partial r$ for $r<a$ and positive for $r>a$ (recall $a$ is the radius of the location of the black circle in Fig. 6). Because $\psi$ is negative, this means $I$ is positive for $z>0$ and negative for $z<0$ consistent with the electric current directions in Fig. 6. The right-hand side of equation (103) acts as a sink for positive angular momentum for $r<a$ and a source for $r>a$. Angular momentum will thus be transferred via the electric circuit from $r<a$ to $r>a$. This shows that any angular momentum removed by the $-r B_{z} J_{r}$ torque from mass at $r<a$ is transferred to mass at $r>a$. Because angular momentum scales as $\rho r U_{\phi}$, only a tiny increment in $U_{\phi}$ at large $r$ is required to absorb the angular momentum removed at small $r$. Very little energy is thus required to transport angular momentum from the $r<a$ region to the $r>a$ region. 
We note that the right-hand side of equation (96) can be expressed as

$\frac{1}{4 \pi^{2}} \nabla \cdot(I \nabla \psi \times \nabla \phi)=\frac{1}{4 \pi^{2}} \nabla \phi \cdot \nabla I \times \nabla \psi$

so sourcing or sinking of angular momentum depends on the angle between $\nabla I$ and $\nabla \psi$. Away from the $z=0$ plane, the poloidal current nearly follows the poloidal flux surfaces so $\nabla I \times \nabla \psi \simeq 0$. However, in the $z=0$ plane $\nabla I \times \nabla \psi \neq 0$ since here the contours of constant $I$ are horizontal whereas the contours of constant $\psi$ are vertical. In the $|z| \gg w$ region, the current accelerates the jet but does not exert a torque. In the $|z| \ll w$ region, this same current transports angular momentum from small $r$ to large $r$ because the direction of $\nabla I$ is the same for all $r$, but the direction of $\nabla \psi$ reverses on going from small to large $r$. Even though minimal torques are involved in the $|z| \gg w$ regions, these regions are necessary for the angular momentum transport in the $|z|<w$ region because they provide a means to complete the circuit for the current. The insulated 'wire' proposed at the end of Section 6 is constituted by the poloidal flux surfaces (i.e. constant $\psi(r, z, t)$ surfaces) in the $|z|>w$ regions because in these regions charged particles are constrained to move on constant poloidal flux surfaces. Upon merging and becoming axisymmetric the spider legs in Fig. 4 correspond to this current-carrying poloidal 'wire'.

The equivalent of the power supply in the lab experiment is the accumulation of the inward spiralling $P_{\phi}=0$ ion/neutral fluid elements (clumps) at small $r$. This inward spiralling tends to cause accumulation of net positive charge at small $r$ in the mid-plane $(+++$ signs in Fig. 6) and neutralizing electrons left behind will cause accumulation of net negative charge at large $r$ in the mid-plane (-- signs in Fig. 6). The positive charge concentration at small $r$ produces axially outward electric fields that will drive axial electric currents perpendicular to the $z=0$ plane at small $r$. These electric currents flow along the arched poloidal flux surfaces to return at large $r$ to the location of the excess electrons in the mid-plane (recall that charged particles are constrained to move on constant $\psi(r, z, t)$ surfaces in the $|z|>w$ region). The current is clockwise in the $z>0$ region and counterclockwise in the $z<0$ region (see the arrows in Fig. 6). Electron flow is opposite to the current direction because electrons are negative. Once all the $P_{\phi}=0$ clumps have spiralled inwards, slower collision processes will create new $P_{\phi}=0$ clumps. Thus, if the $P_{\phi}=0$ clumps are continuously replenished there will be a continuous inflow, continuous electric currents, and continuous jets. If $P_{\phi}$ is not exactly zero, then the clump cannot penetrate exactly to $r=0$ but it only need penetrate to the radius of the jet.

At $z$ altitudes far above or below the accretion disc (i.e. $|z| \gg w$ ), the gas is orders of magnitude more diffuse than in the disc so the local particle collision frequency $v=\sigma n v_{\mathrm{T}}$ becomes smaller than the electron and ion cyclotron frequencies. Charged particles now execute complete cyclotron orbits and ideal MHD becomes valid. Thus, the Hall MHD region in the accretion disc constitutes a gravity-powered dynamo that drives currents in the ideal MHD region above and below the disc. Numerical models based on ideal MHD will not capture the dynamo behaviour in the $|z|<w$ region.

The ionization fraction is very small in the disc (i.e. region near the $z=0$ plane) and has values in the range $10^{-13}-10^{-8}$ whereas the ionization fraction is near-unity in the ideal MHD region at altitudes well above and below $z=0$. As $|z|$ increases, $\alpha$ will increase from being $10^{-13}-10^{-8}$ to being of order unity and so there will always be an altitude $z$ where $P_{\phi}=0$ for an ion/neutral clump. The $P_{\phi}=0$ clump will spiral inwards with a fast radial velocity, essentially the free-fall velocity. The degree of ionization of a clump will also likely change with time because (i) the distance between a clump and the source of ionization changes, (ii) something temporarily shields the clump from the source of ionization or (iii) the source of ionization changes. A clump may have epicyclic motion in the $z$-direction so its $\alpha$ would oscillate, being lower as it approached $z=0$ and higher as it swung to higher $z$. For all these reasons, it is reasonable to expect that clumps can have $P_{\phi}=0$ for at least some of the time. Since a clump can never spiral outwards, the cumulative effect of the times when a clump has $P_{\phi}=0$ and effectively spirals inwards will simply reduce the accretion rate to be slower than the free-fall velocity. Also, viscosity although very small is not zero, so on the very long time-scale adjacent fluid elements will exchange angular momentum via viscous drag; this will cause a slow variation in $P_{\phi}$ of fluid elements so that they may go from having finite $P_{\phi}$ to having zero $P_{\phi}$.

These falling positively charged clumps constitute a radially inward electric current in the $z=0$ plane. This electric current is part of a global electric circuit where the remaining part of the circuit is comprised of the motion of electrons and ions along poloidal field lines in the ideal MHD regions located above and below the $z=0$ plane. These poloidal currents have topology identical to that of the Caltech jet experiment and the $\boldsymbol{J} \times \boldsymbol{B}$ force associated with these currents drives bidirectional astrophysical jets that flow along the $z$-axis away from the $z=0$ plane. The jet can be roughly considered as resulting from an inflation of the poloidal flux surfaces by the pressure of the toroidal field produced by the poloidal current, i.e. the magnetic tower mechanism (Lynden-Bell 2003). The particle source for these jets is the infalling $P_{\phi}=0$ ion/neutral clumps so the infalling $P_{\phi}=0$ ion/neutral clumps are both the equivalent of the power supply in the Caltech experiment (in the clump case the power comes from gravity) and the equivalent of the gas source in the Caltech experiment.

The $P_{\phi}=0$ situation is like having a bucket with a triangular hole in its bottom containing triangular coins that just fit through the hole so a coin can fall through the hole only when correctly aligned. If the bucket is shaken, then the coins will assume arbitrary alignments over time and at any time a few coins will be aligned to fall through the hole. After a long time, all the coins will fall through, i.e. all clumps will eventually experience enough $P_{\phi}=0$ intervals that they fall to the central object. Since having $P_{\phi}=0$ depends on the properties of a velocity component, the drainage of $P_{\phi}=0$ particles from the overall volume to the vicinity of the central object is to some extent analogous to the loss-cone of a magnetic mirror, a situation where leakage from the system depends on velocity properties. A magnetic mirror has two regions of strong magnetic field $B_{\max }$ separated by a region of weak magnetic field $B_{\text {min }}$ and particles having a certain range of velocity angles are trapped in the weak-field region. Magnetic mirror loss-cones are discussed in the general plasma context on pages 34-35 of Chen (1984), in the astrophysical context on p. 25 of Kulsrud (2005), in the magnetospheric context by Tsurutani \& Lakhina (1997), and in the solar corona context by Aschwanden (1998). In a magnetic mirror, only particles having $v_{\perp}^{2} / v^{2}$ greater than the mirror ratio $B_{\min } / B_{\max }$ are trapped, where $v_{\perp}$ denotes the velocity component perpendicular to the magnetic field. Particles with $v_{\perp}^{2} / v^{2}$ less than the mirror ratio escape and are said to be in the loss cone. Collisions can alter $v_{\perp}^{2} / v^{2}$ (i.e. change the pitch angle) and so can scatter a particle into the loss cone. Thus, particle leakage from the magnetic mirror results from collisions changing the velocity pitch angle. Similarly, over long times collisions could change $P_{\phi}$ of a clump so that $P_{\phi}$ changes from non-zero to zero allowing the 
particle to escape from the volume and drain towards the central object.

This accretion of clumps with $P_{\phi}=0$ in the $z=0$ plane violates equation (1) because $U_{r}$ and $B_{z}$ are finite whereas $U_{z}=0, B_{r}=0$ and $E_{\phi}=0$ so $E_{\phi}+U_{z} B_{r}-U_{r} B_{z} \neq 0$. Outside the $z=0$ plane where collisions are negligible, electrons and ions are magnetized and restricted to move along surfaces of constant $\psi$. In contrast, ions in the $z=0$ plane are unmagnetized and a component of the clumps; the $P_{\phi}=0$ clumps can move across the surfaces of constant $\psi$. The $P_{\phi}=0$ clump motion in the $z=0$ plane constitutes an electrical power source because gravity is forcing the radial inward clump motion (see discussion after equation 58); the $z=0$ plane can equivalently be seen to be an electric power source because $\boldsymbol{J} \cdot \boldsymbol{E}$ is negative in the $z=0$ plane as is the situation inside a battery that is driving a load. The jet motion outside the $z=0$ plane is an electrical power sink because the jets are being accelerated by forces resulting from the current in this circuit; the region outside the $z=0$ plane is seen to be an electric power sink (or load) because in this region $\boldsymbol{J} \cdot \boldsymbol{E}$ is positive as is the case inside a load being driven by a battery.

This argument is further validated from consideration of the Hall Ohm's law

$\boldsymbol{E}+\boldsymbol{U} \times \boldsymbol{B}-\frac{\boldsymbol{J}}{n_{\mathrm{e}} e} \times \boldsymbol{B}+\frac{1}{n_{\mathrm{e}} e} \nabla P_{\mathrm{e}} \simeq 0$.

The electric current density is

$\boldsymbol{J}=n_{\mathrm{e}} e\left(\boldsymbol{u}_{\mathrm{i}}-\boldsymbol{u}_{\mathrm{e}}\right) \simeq n_{\mathrm{e}} e\left(\boldsymbol{U}-\boldsymbol{u}_{\mathrm{e}}\right)$

so the azimuthal component of equation (105) becomes

$E_{\phi}+U_{z} B_{r}-U_{r} B_{z}-\frac{1}{n_{\mathrm{e}} e}\left(J_{z} B_{r}-J_{r} B_{z}\right) \simeq 0$.

In the $z=0$ plane where $E_{\phi}=0, U_{z}=0, B_{r}=0$ and $J_{z}=0$, equation (107) reduces to

$-U_{r} B_{z}+\frac{1}{n_{\mathrm{i}} e} J_{r} B_{z} \simeq 0$

or

$J_{r} \simeq n_{\mathrm{e}} e U_{r}$

so as discussed, there is a radially inward current carried by the positive clumps and there is a radially outward electric field in the $z=0$ plane so the $z=0$ plane acts like a battery that is driving the current in the load outside of the $z=0$ plane.

\section{ORDER OF MAGNITUDE QUANTITATIVE ANALYSIS}

\subsection{Overview and results}

This section presents order of magnitude quantitative estimates for various relevant parameters. These estimates are obtained by combining constraints provided by disc/jet observational properties with results from the previous sections. These considerations provide the following constraints for a one solar mass star, i.e. $M=\mathrm{M}_{\odot}$ :

(i) The accretion rate is $\dot{M}=10^{-9}-10^{-7} \mathrm{M}_{\odot} \mathrm{yr}^{-1}$.

(ii) The jet kinetic energy density and magnetic energy density are approximately equal at the base of the jet so the jet energy density is $\rho_{\text {jet }} U_{\text {jet }}^{2}$ and the jet energy flux is thus $\rho_{\text {jet }} U_{\text {jet }}^{3}$.

(iii) The ratio of jet mass flow to disc accretion (ejection/accretion ratio) is $\varepsilon_{\mathrm{m}}=0.01-0.07$ (Coffey, Bacciotti \& Podio 2008).
Table 1. Assumed inputs for all quantitative estimates.

\begin{tabular}{llll}
\hline Nominal input parameter & Symbol & Value & Units \\
\hline Jet velocity & $U_{\text {jet }}$ & $2 \times 10^{5}$ & $\mathrm{~m} \mathrm{~s}^{-1}$ \\
Mass accretion rate & $\dot{M}$ & $10^{-8}$ & $\mathrm{M} \mathrm{yr}^{-1}$ \\
Jet to disc power ratio & $\varepsilon_{\mathrm{p}}$ & 0.5 & \\
Mass ejection to accretion ratio & $\varepsilon_{\mathrm{m}}$ & 0.03 & \\
\hline
\end{tabular}

(iv) The nominal jet velocity is $U_{\text {jet }}=2 \times 10^{5} \mathrm{~m} \mathrm{~s}^{-1}$ (Coffey et al. 2008).

(v) The electric current flowing in the disc flows along bidirectional jets as in Fig. 6 and drives these jets as described in Hsu \& Bellan (2002), You et al. (2005), Kumar \& Bellan (2009) and Zhai et al. (2014).

For simplicity, it is assumed that the accreting material, ions, and jet material are all hydrogen with $m_{\mathrm{n}}=m_{\mathrm{i}}$ so $\alpha$ is the ionization fraction. More exacting analysis would distinguish between atoms and molecules as well as take into account masses of different species.

We assume the following nominal values as inputs: $U_{\text {jet }}=2 \times$ $10^{5} \mathrm{~m} \mathrm{~s}^{-1}, \dot{M}=10^{-8} \mathrm{M}_{\odot} \mathrm{yr}^{-1}$, and $\varepsilon_{\mathrm{m}}=0.03$; for reference these input parameters are also listed in Table 1 . The analysis below calculates various quantities using these input parameters. Table 2 lists these calculated parameters and cites the equations used for each calculation. The results are presented both in SI units and in units commonly used in astrophysics.

\subsection{Derivation of the quantitative estimates}

The accretion power $P_{\text {acc }}$ is the gravitational potential energy per second released by accreting matter on coming from infinity to the jet radius $r_{\text {jet }}$ and is

$P_{\mathrm{acc}}=\frac{M \dot{M} G}{r_{\mathrm{jet}}}$.

Using item (ii) in the above list the jet power (both jets, including kinetic and magnetic energy content) is

$P_{\text {jet }}=2 \rho_{\text {jet }} U_{\text {jet }}^{3} \pi r_{\text {jet }}^{2}$.

Defining $\varepsilon_{\mathrm{p}}$ to be the fraction of accretion power going into the jets, the jet power is related to the accretion power by

$2 \rho_{\mathrm{jet}} U_{\mathrm{jet}}^{3} \pi r_{\mathrm{jet}}^{2}=\varepsilon_{\mathrm{p}} \frac{M \dot{M} G}{r_{\mathrm{jet}}}$

it will later be shown that $\varepsilon_{\mathrm{p}}=0.5$ and so this value will be used for quantitative estimates. From item (iii) on the list of constraints, the accretion mass flux is

$2 \rho_{\text {jet }} U_{\text {jet }} \pi r_{\text {jet }}^{2}=\varepsilon_{\mathrm{m}} \dot{M}$

where the left-hand side is the rate of mass ejection of the two jets.

Dividing equation (112) by equation (113) and solving for $U_{\text {jet }}$ gives

$U_{\text {jet }}=\sqrt{\frac{\varepsilon_{\mathrm{p}}}{\varepsilon_{\mathrm{m}}} \frac{M G}{r_{\mathrm{jet}}}}$.

The jet axial starting position is assumed to be the same as the jet radius. Since the escape velocity at this location is $\sqrt{2 M G / r_{\text {jet }}}$ a necessary requirement for the jet to launch is that $\varepsilon_{\mathrm{p}} / \varepsilon_{\mathrm{m}}>2$ which is well satisfied by the assumed values for $\varepsilon_{\mathrm{p}}, \varepsilon_{\mathrm{m}}$. 
Table 2. Calculated quantities from model in SI and cgs units, distances in au; reference is given to relevant equations used.

\begin{tabular}{|c|c|c|c|c|c|c|}
\hline Nominal calculated quantity & Symbol & SI Value & SI Units & Reference & Value & Units \\
\hline Jet power (both jets) & $P_{\text {jet }}$ & $7.6 \times 10^{23}$ & $\mathrm{~W}$ & Equation (111) & $7.6 \times 10^{30}$ & $\operatorname{erg~s}^{-1}$ \\
\hline Jet mass density & $\rho_{\text {jet }}$ & $4.9 \times 10^{-15}$ & $\mathrm{~kg} \mathrm{~m}^{-3}$ & Equation (116) & $4.9 \times 10^{-18}$ & $\mathrm{~g} \mathrm{~cm}^{-3}$ \\
\hline Jet density & $n_{\text {jet }}$ & $2.9 \times 10^{12}$ & $m^{-3}$ & Equation (117) & $2.9 \times 10^{6}$ & $\mathrm{~cm}^{-3}$ \\
\hline Disc ionization fraction & $\alpha$ & $2.9 \times 10^{-10}$ & & Equation (128) & $2.9 \times 10^{-10}$ & \\
\hline Disc electric current & $I_{\text {disc }}$ & $1.8 \times 10^{13}$ & A & Equation (129) & $5.3 \times 10^{22}$ & statamperes \\
\hline Toroidal magnetic field at $r_{\text {nom }}$ & $B_{\phi}$ & $7.9 \times 10^{-6}$ & $\mathrm{~T}$ & Equation (130) & 79 & milligauss \\
\hline Voltage driving jets & $V_{\text {jet }}$ & $8.6 \times 10^{10}$ & $\mathrm{~V}$ & Equation (132) & $2.9 \times 10^{8}$ & statvolts \\
\hline Disc internal resistance & $R_{\mathrm{disc}}$ & $2.4 \times 10^{-3}$ & ohm & Equation (135) & $2.7 \times 10^{-15}$ & $\mathrm{~s} \mathrm{~cm}^{-1}$ \\
\hline Disc neutral density at $r_{\text {nom }}$ & $n_{\mathrm{n}}$ & $6.0 \times 10^{19}$ & $\mathrm{~m}^{-3}$ & Equation (151) & $6.0 \times 10^{13}$ & $\mathrm{~cm}^{-3}$ \\
\hline Fractional thickness of $P_{\phi}=0$ layer & $\varepsilon_{\mathrm{c}}$ & $10^{-7}$ & & Equation (148) & $10^{-7}$ & \\
\hline Disc temperature at $r_{\text {nom }}$ & $T$ & 48 & $\mathrm{~K}$ & Equation (149) & 48 & $\mathrm{~K}$ \\
\hline Ion-neutral binding condition & $v_{\mathrm{in}} / \omega_{\mathrm{ci}}$ & $1.5 \times 10^{2}$ & & Equation (154) & $1.5 \times 10^{2}$ & \\
\hline
\end{tabular}

Solving equation (114) for $r_{\text {jet }}$ gives

$r_{\mathrm{jet}}=\frac{\varepsilon_{\mathrm{p}}}{\varepsilon_{\mathrm{m}}} \frac{M G}{U_{\mathrm{jet}}^{2}}$.

Inserting $r_{\text {jet }}$ and $U_{\text {jet }}$ in equation (113) gives

$\rho_{\mathrm{jet}}=\frac{\varepsilon_{\mathrm{m}}^{3} \dot{M} U_{\mathrm{jet}}^{3}}{2 \pi \varepsilon_{\mathrm{p}}^{2} M^{2} G^{2}}$

corresponding to a fully ionized jet density

$n_{\text {jet }}=\frac{\rho_{\text {jet }}}{m_{\mathrm{i}}}$

so as seen from Table 2 the jet is much more diffuse than the accretion disc.

The number of particles accreting per second is $\dot{M} / m_{\mathrm{n}}$ and each carries a nominal charge $\alpha e$. The electric current flowing in the disc resulting from accretion then is

$I_{\text {disc }}=\frac{\dot{M}}{m_{\mathrm{n}}} \alpha e ;$

half of this current flows in each of the jets as shown in Fig. 6. If there was no completion of the electrical circuit through the jets, positive charge would accumulate at small radius until the opencircuit electric field given by equation (88) would develop and inhibit further accumulation. There would thus be a potential drop, but no electric current. The disc has electrical resistivity because of electron-neutral collisions and the system with no current would look like Fig. 7(a). The distributed nature of the resistivity and of the gravitationally produced electric field is indicated by Fig. 7(a) having several resistors and several batteries in series. The situation in Fig. 7(a) is electrically equivalent to the situation in Fig. 7(b) where $V_{\text {open cct }}$ indicates the integral from the jet radius to infinity of the electric field given in equation (88) and $R_{\text {disc }}$ is the sum of the distributed resistances and can be considered the internal resistance of the disc. The open-circuit voltage is the same as in (a). Fig. 7(c) shows the circuit when the disc is connected to the bidirectional jets as in Fig. 6. Half the disc current goes to the upper jet and half to the lower jet. The electrical loading by each jet is represented by a resistance $R_{\text {jet }}$. (a)

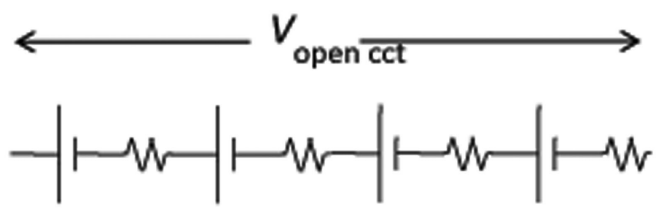

(b)

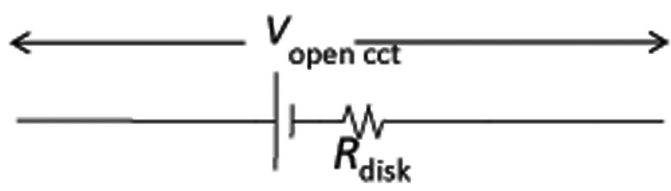

(c)

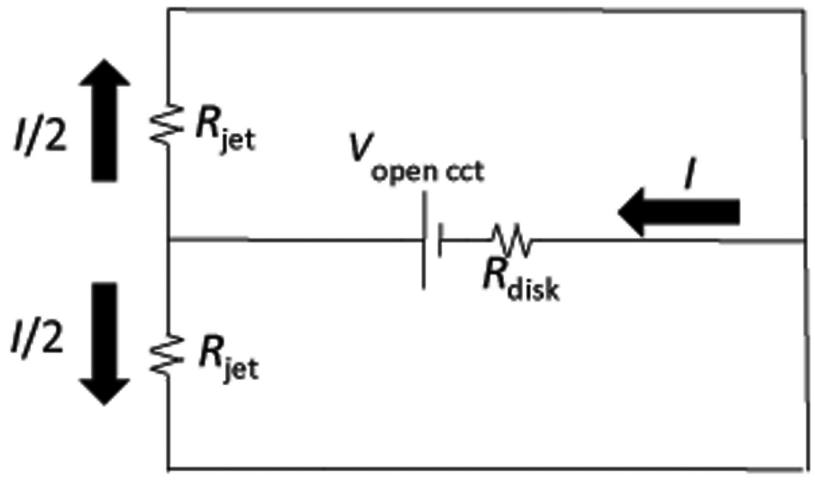

Figure 7. (a) Open-circuit situation: disc can be considered as a chain of batteries with each battery having an internal resistance; (b) this is equivalent to one large battery and one large resistor; (c) disc battery and resistor connected to bidirectional jets where each jet has an effective load resistance $R_{\text {jet }}$. 
Because the two jets are electrically in parallel, the load resistance presented to the accretion disc dynamo is $R_{\text {load }}=R_{\text {jet }} / 2$. The electric circuit in Fig. 7(c) can be expressed as

$V_{\text {open cct }}=I\left(R_{\text {disc }}+R_{\text {load }}\right)$,

which is the same as a battery having open-circuit voltage $V_{\text {opencct }}$ and internal resistance $R_{\text {disc }}$ connected to a load with resistance $R_{\text {load }}$. The power coupled to the jets is

$$
\begin{aligned}
P_{\text {jet }} & =I^{2} R_{\text {load }} \\
& =\frac{R_{\text {load }} / R_{\text {disc }}}{\left(1+\frac{R_{\text {load }}}{R_{\text {disc }}}\right)^{2}} \frac{V_{\text {open cct }}^{2}}{R_{\text {disc }}},
\end{aligned}
$$

which is at a maximum when $R_{\text {load }}=R_{\text {disc }}$, the well-known impedance matching condition. In this case $I^{2} R_{\text {disc }}=I^{2} R_{\text {load }}$ so the power going into the jets equals the power internally dissipated in the accretion disc. If $R_{\text {load }}=R_{\text {disc }}$, then the voltage drop across the disc internal resistance is half the open-circuit voltage and so the electric field in the disc will be half the open-circuit electric field given by equation (88). Thus under matched load conditions $R_{\text {load }}=R_{\text {disc }}$ the electric field in the disc is

$E_{r}^{\text {loaded }}=\frac{E_{r}^{\text {Kepler }}}{2}$

using equation (88). This corresponds to setting $\beta=1 / 2$ in equation (20). Fig. 1(h) shows that in this $\beta=1 / 2$ situation particles with $P_{\phi}=0$ will spiral down towards $r=0$.

Since $E_{r}^{\text {Kepler }}=-U_{\phi} B_{z}$, this gives

$\frac{E_{r}^{\text {loaded }}}{B_{z}}=-\frac{U_{\phi}}{2}$

and so equations (71), the solution to the generalized Ohm's law equation (66), give

$u_{e r} \simeq-\frac{\kappa}{2} U_{\phi}$

$u_{e \phi} \simeq \frac{U_{\phi}}{2}$,

where we have used the fact that $U_{r}$ is of the order of $U_{\phi}$ or smaller. Since $\kappa=v_{\text {en }} / \omega_{\text {ce }} \ll 1$ electrons have negligible radial motion and so are frozen to poloidal flux surfaces. Unlike the ideal Ohm's law equation (19) which is solved to give the radial electric field, the solution to the generalized Ohm's law consists of the electron fluid velocities.

We now assume that the system operates in this maximum power transfer mode and will justify this assumption at the end of this section. Thus, half the accretion power is dissipated in the disc while the remaining half goes into driving the jets. Each jet would then absorb one quarter of the gravitational energy released by accreting mass (accretion power).

Fig. 8 in Zhai et al. (2014) indicates that a typical jet has $\left|B_{z}\right|=\left|B_{\phi}\right|$ at a nominal radius $r_{\text {nom }} \simeq 4 r_{\text {jet }}$; this location can be considered to be the outer extent of the jet and the inner radius of the accretion disc. The $\left|B_{z}\right|=\left|B_{\phi}\right|$ relation corresponds to the pressure of the toroidal magnetic field being sufficiently strong to overcome the 'field line tension' associated with the poloidal field and so generate a flow. This condition can equivalently be seen as the spheromak formation condition $\mu_{0} I>\lambda \psi$, where $B_{\phi}=\mu_{0} I_{\text {jet }} /(2 \pi r), \psi=B_{z} \pi r^{2}$ and $\lambda \sim 2 / r$ (Bellan 2000).
From Ampere's law and using $I_{\text {jet }}=I_{\text {disc }} / 2$ and equation (118), the toroidal field is

$B_{\phi} \simeq \frac{\mu_{0} I_{\mathrm{jet}}}{2 \pi r}=\frac{\mu_{0}}{4 \pi r} \frac{\dot{M}}{m_{\mathrm{n}}} \alpha e$.

From equation (60), the $P_{\phi}=0$ condition implies

$\dot{\phi}=-\frac{\alpha e}{2 m_{\mathrm{n}}} B_{z}$

while the presumption that the angular velocity of the $P_{\phi}=0$ particles is approximately Keplerian implies $\dot{\phi} \simeq \sqrt{M G / r^{3}}$ so

$B_{z} \simeq-\frac{2 m_{\mathrm{n}}}{\alpha e} \sqrt{\frac{M G}{r^{3}}}$

Setting $\left|B_{z}\right|=\left|B_{\phi}\right|$ at $r \simeq 4 r_{\text {jet }}$ gives

$\frac{\mu_{0}}{4 \pi r} \frac{\dot{M}}{m_{\mathrm{n}}} \alpha e=\frac{2 m_{\mathrm{n}}}{\alpha e} \sqrt{\frac{M G}{r^{3}}}$

which can be solved for $\alpha$ at $r_{\text {nom }}=4 r_{\text {jet }}$ using equation (115) to give

$\alpha=\frac{m_{\mathrm{n}}}{e} \sqrt{\frac{4 \pi U_{\text {jet }}}{\mu_{0} \dot{M}}}\left(\frac{\varepsilon_{\mathrm{m}}}{\varepsilon_{\mathrm{p}}}\right)^{1 / 4}$.

Because of the $1 / 4$ power in equation (128) equating $\left|B_{z}\right|=\left|B_{\phi}\right|$ at a different radius than $4 r_{\text {jet }}$ would make negligible difference to the value of $\alpha$; for example, using $8 r_{\text {jet }}$ instead of $4 r_{\text {jet }}$ would change $\alpha$ by less than 20 per cent.

Substituting for $\alpha$ in equation (118) gives

$I_{\mathrm{disc}}=\sqrt{\frac{4 \pi \dot{M} U_{\mathrm{jet}}}{\mu_{0}}}\left(\frac{\varepsilon_{\mathrm{m}}}{\varepsilon_{\mathrm{p}}}\right)^{1 / 4}$.

This electric current is carried by the $P_{\phi}=0$ particles which exist in a thin layer of the disc where $\alpha$ is as given by equation (128). This layer will be somewhere between the disc surface (where $\alpha=1$ since the plasma external to the disc is fully ionized) and $z=0$ (vicinity of dead zone regions where $\alpha=0$ in fig. 1 of Gammie (1996). In contrast to MRI models which are adversely affected by dead zones, the model presented here is not only unaffected by their existence but in fact, depends on there being a stratification of $\alpha$ so that $\alpha$ assumes all values from zero to unity in moving vertically from $z=0$ (disc mid-plane) to outside the disc (fully ionized plasma).

At $r_{\text {nom }}=4 r_{\text {jet }}$, the toroidal magnetic field is given by $B_{\phi}=\mu_{0} I_{\text {jet }} /\left(2 \pi \times 4 r_{\text {jet }}\right)=\mu_{0} I_{\text {disc }} /\left(2 \pi \times 4 r_{\text {jet }} \times 2\right)$. Using equation (129) for $I_{\text {disc }}$ and equation (115), this toroidal field at $r_{\text {nom }}$ is

$\left|B_{\phi}\right|=\frac{U_{\text {jet }}^{5 / 2}}{8 \pi M G} \sqrt{\pi \mu_{0} \dot{M}}\left(\frac{\varepsilon_{\mathrm{m}}}{\varepsilon_{\mathrm{p}}}\right)^{5 / 4} \mathrm{~T}$.

At larger radii, $B_{\phi}$ would fall off from this value as $r^{-1}$.

Using equations (116) and (115) in equation (111), the jet power (both jets) is

$P_{\text {jet }}=\varepsilon_{\mathrm{m}} \dot{M} U_{\text {jet }}^{2}$

and the voltage driving the two jets is

$V_{\text {jet }}=\frac{P_{\text {jet }}}{I_{\text {disc }}}$.

The open-circuit voltage obtained by integrating equation (88) from infinity to the jet radius is

$V_{\text {open }}=-\int_{\infty}^{r_{\text {jet }}} \frac{2 m_{\mathrm{i}} M G}{\alpha e r^{2}} \mathrm{~d} r=\frac{2 m_{\mathrm{i}} M G}{\alpha e r_{\mathrm{jet}}}$. 
Because of the assumption of maximum coupling of electrical power from the disc to the jets, the voltage driving the jets is half the opencircuit voltage, i.e.

$V_{\mathrm{jet}}=\frac{m_{\mathrm{i}} M G}{\alpha e r_{\mathrm{jet}}}$.

Because the jet power is half the accretion power, the remaining half of the accretion power must be dissipated in the accretion disc itself, in analogy to a battery getting warm when powering a load. This dissipation results from the frictional drag experienced by the metaparticles colliding with electrons, i.e. from the effective electrical resistivity. Since the power dissipated in the disc equals the power absorbed by the jets, using equations (129) and (131) gives

$R_{\mathrm{disc}}=\frac{P_{\mathrm{disc}}}{I_{\mathrm{disc}}^{2}}=\frac{P_{\mathrm{jet}}}{I_{\mathrm{disc}}^{2}}=\frac{\mu_{0} \sqrt{\varepsilon_{\mathrm{m}} \varepsilon_{\mathrm{p}}} U_{\mathrm{jet}}}{4 \pi} \mathrm{ohm}$.

This resistance does not correspond to what would be calculated using a bulk resistivity integrated over the entire disc volume because the disc current flows only in a thin disc layer for which $\alpha$ is given by equation (128). In particular, the dead zones near $z=0$ do not interrupt the current because the current does not flow in the dead zones.

A necessary condition for this model is that electrons should be frozen into poloidal flux surfaces; this occurs if the electron cyclotron frequency exceeds the electron-neutral collision frequency $v_{\mathrm{en}}=n_{\mathrm{n}}\langle\sigma v\rangle_{\mathrm{en}}$, i.e. if

$\frac{e\left|B_{z}\right|}{m_{\mathrm{e}}}>n_{\mathrm{n}}\langle\sigma v\rangle_{\mathrm{en}}$

where the electron-neutral collision rate coefficient is (Balbus \& Terquem 2001)

$\langle\sigma v\rangle_{\mathrm{en}}=8.3 \times 10^{-16} T^{1 / 2} \mathrm{~m}^{3} \mathrm{~s}^{-1}$.

Since the open-circuit electric field balances the sum of the last two terms in equation (86) and since these last two terms are equal in magnitude for $\alpha \omega_{\mathrm{ci}}=-2 \dot{\phi}$, the loaded electric field is equal to one of the last two terms. The radial centre-of-mass velocity of the $P_{\phi}=0$ fluid element in the jet-loaded disc will then be $U_{r} \simeq$ $-\sqrt{M G / r}$. The $P_{\phi}=0$ condition gives the toroidal ion and neutral velocity to be $U_{\phi}=-\alpha r e B_{z} / m_{\mathrm{i}} \approx \sqrt{M G / r}$ and it is recalled that $B_{z}$ is negative.

Because $U_{r} \simeq-\sqrt{M G / r}$ the radial current density is

$J_{r}=n_{\mathrm{i}} e U_{r}=-\alpha n_{\mathrm{n}} e \sqrt{M G / r}$.

We denote the thickness of the $P_{\phi}=0$ infalling layer to be $\varepsilon_{\mathrm{c}} r$ where $\varepsilon_{\mathrm{c}}$ is to be determined. The disc current density can then be calculated from the accretion electric current equation (118) to be

$J_{r}=-\frac{I_{\mathrm{disc}}}{2 \pi \varepsilon_{\mathrm{c}} r^{2}}=-\frac{\dot{M} \alpha e}{2 \pi \varepsilon_{\mathrm{c}} m_{\mathrm{n}} r^{2}}$.

Equating the current densities given by equations (138) and (139) gives

$n_{\mathrm{n}}=\frac{\dot{M}}{2 \pi \varepsilon_{\mathrm{c}} m_{\mathrm{n}} \sqrt{M G r^{3}}}$.

The load matching resulted in half the accretion power $P_{\text {acc }}$ being dissipated in the disc and half in the jets, i.e. $I^{2} R_{\text {disc }}=P_{\text {acc }} / 2$. This relation can be expressed in terms of power ohmically dissipated in the disc and the gravitational energy liberated as

$\eta J^{2}=\frac{1}{2} \frac{\rho M G}{r^{2}}\left|U_{r}\right|$, where the electrical resistivity is

$\eta=\frac{m_{\mathrm{e}} v_{\mathrm{en}}}{n_{\mathrm{e}} e^{2}}=\frac{m_{\mathrm{e}} n_{\mathrm{n}}\langle\sigma v\rangle_{\mathrm{en}}}{n_{\mathrm{e}} e^{2}}=\frac{m_{\mathrm{e}}\langle\sigma v\rangle_{\mathrm{en}}}{\alpha e^{2}}$.

Because the azimuthal ion speed is $U_{\phi}$ and the azimuthal electron speed is $U_{\phi} / 2$, the azimuthal current is $J_{\phi}=n_{\mathrm{e}} e U_{\phi} / 2$. In contrast, the radial ion speed is of the order of $U_{\phi}$ while the radial electron speed is nearly zero. Thus, $J_{r}^{2} \gg J_{\phi}^{2}$ and so $J^{2} \simeq J_{r}^{2}$. Equation (141) can be written using equation (142) as

$\frac{m_{\mathrm{e}}\langle\sigma v\rangle_{e N}}{\alpha e^{2}}=\frac{1}{2} \frac{\rho M G}{J_{r}^{2} r^{2}}\left|U_{r}\right|$

Substituting for $J_{r}$ using equation (138) gives

$v_{\mathrm{en}}=n_{\mathrm{n}}\langle\sigma v\rangle_{e N}=\frac{1}{2} \frac{\rho\left|U_{r}\right|}{\alpha n_{\mathrm{n}} m_{\mathrm{e}} r}=\frac{1}{2} \frac{m_{\mathrm{i}}\left|U_{r}\right|}{\alpha m_{\mathrm{e}} r}$.

Thus,

$\kappa=\frac{v_{\mathrm{en}} m_{\mathrm{e}}}{e B_{z}}=\frac{1}{2} \frac{\left|U_{r}\right|}{\alpha \omega_{\mathrm{ci}} r}$

However, $\left|U_{r}\right| \simeq \sqrt{M G / r}=U_{\phi}=r|\dot{\phi}|$ and $|\dot{\phi}|=\alpha\left|\omega_{\mathrm{ci}}\right| / 2$ for $P_{\phi}=0$ so

$\kappa \simeq-\frac{1}{4}$

noting that $B_{z}$ is negative. Thus, the condition that maximum power is coupled from the accretion disc to the jets (i.e. $I^{2} R_{\mathrm{disc}}=P_{\mathrm{acc}} / 2$ ) causes the configuration to be in the Hall regime, i.e. satisfy the assumption that $|\kappa| \ll 1$.

Because this is a steady-state model, the power dissipated in the disc must be radiated away as blackbody radiation. Consider the annular disc segment of the $P_{\phi}=0$ layer between $r$ and $r+\mathrm{d} r$. The top and bottom of this annular segment each have surface area $2 \pi r \mathrm{~d} r$ and the segment height is $\varepsilon_{\mathrm{c}} r$ so the segment volume is $2 \pi \varepsilon_{\mathrm{c}} r^{2} \mathrm{~d} r$. The power radiated from the segment top and bottom surfaces is $\mathrm{d} P_{\text {rad }}=\sigma_{\mathrm{SB}} T^{4} 4 \pi r \mathrm{~d} r$, where $\sigma_{\mathrm{SB}}=5.7 \times 10^{-8} \mathrm{~W} \mathrm{~m}^{-2} \mathrm{~K}^{-4}$ is the Stefan-Boltzmann blackbody radiation coefficient. The ohmic power dissipated in the segment volume $2 \pi \varepsilon_{\mathrm{c}} r^{2} \mathrm{~d} r$ is $\mathrm{d} P_{\text {ohmic }}=$ $\eta J^{2} 2 \pi \varepsilon_{\mathrm{c}} r^{2} \mathrm{~d} r$. In equilibrium the radiated power equals the ohmic dissipation, i.e.

$2 \sigma_{\mathrm{SB}} T^{4}=\eta J^{2} \varepsilon_{\mathrm{c}} r$.

The radial mass flux through the layer of height $\varepsilon_{\mathrm{c}} r$ at radial location $r$ is $\dot{M}=\rho U_{r} \times 2 \pi r \times \varepsilon_{\mathrm{c}} r$ so

$\varepsilon_{\mathrm{c}}=\frac{\dot{M}}{\rho U_{r} 2 \pi \varepsilon_{\mathrm{c}} r^{2}}$.

Substituting for $\varepsilon_{\mathrm{c}}$ and $\eta J^{2}$ in equation (147) using equations (148) and (141), respectively, gives

$T=\left(\frac{\dot{M} M G}{8 \pi \sigma_{\mathrm{SB}} r^{3}}\right)^{1 / 4} \mathrm{~K}$,

which gives $T=48 \mathrm{~K}$ for the accretion rate in Table 1 and $r=r_{\text {nom. }}$.

The Hall condition can then be used to specify a constraint on the density since equation (146) implies $n_{\mathrm{n}}\langle\sigma v\rangle_{\mathrm{en}} / \omega_{\mathrm{ce}}=1 / 4$ so

$n_{\mathrm{n}}=\frac{\omega_{\mathrm{ce}}}{4\langle\sigma v\rangle_{\mathrm{en}}}$. 
Using $\left|B_{z}\right|=\left|B_{\phi}\right|$, equations (130), (137) and (149), this becomes

$$
\begin{aligned}
n_{\mathrm{n}}= & \frac{1}{4 \times 8.3 \times 10^{-16}}\left(\frac{8 \pi \sigma_{\mathrm{SB}} r^{3}}{\dot{M} M G}\right)^{1 / 8} \\
& \times \frac{e}{m_{\mathrm{e}}} \frac{U_{\mathrm{jet}}^{5 / 2}}{8 \pi M G} \sqrt{\pi \mu_{0} \dot{M}}\left(\frac{\varepsilon_{\mathrm{m}}}{\varepsilon_{\mathrm{p}}}\right)^{5 / 4} \mathrm{~m}^{-3} .
\end{aligned}
$$

We now justify the assumption that the configuration arranges itself so that the jet power equals the power dissipated in the accretion disc (i.e. $\varepsilon_{\mathrm{p}}=0.5$ ), the situation of maximum power transfer. Let $T_{\text {eq }}$ be the disc temperature at which the power lost via by blackbody radiation equals the ohmic power dissipated in the disc when the load resistance $R_{\text {load }}$ provided by the jets equals the disc internal resistance $R_{\text {disc }}$. Thus at $T_{\text {eq }}$ the radiated power is $\dot{M} M G /(2 r)$ since the ohmic power is half the accretion power. Since the radiated power scales as $T^{4}$ and since an excess of ohmic heating power over radiated power causes disc heating,

$$
\begin{aligned}
\frac{\mathrm{d} T}{\mathrm{~d} t} & \sim \frac{\dot{M} M G}{r} \frac{R_{\mathrm{disc}}}{R_{\mathrm{disc}}+R_{\mathrm{load}}}-\frac{\dot{M} M G}{2 r}\left(\frac{T}{T_{\mathrm{eq}}}\right)^{4} \\
& =\frac{\dot{M} M G}{r}\left(\frac{\xi^{1 / 2}}{\xi^{1 / 2}+1}-\frac{1}{2} \xi^{4}\right),
\end{aligned}
$$

where $\xi=T / T_{\text {eq }}$ and $R_{\text {disc }} / R_{\text {load }} \sim \xi^{1 / 2}$ have been used. Equation (152) shows that the temperature increases if $\xi<1$ and decreases if $\xi>1$ showing that the temperature will equilibrate at $\xi=1$ and so tend towards the $R_{\text {disc }}=R_{\text {load }}$ situation.

The calculated density, temperature, and magnetic field at $r_{\text {nom }}$ appear quite reasonable; Table 2 lists these as $n_{\mathrm{n}}=6 \times 10^{19} \mathrm{~m}^{-3}=$ $6 \times 10^{13} \mathrm{~cm}^{-3}, T=48 \mathrm{~K}$ and $B=7.9 \times 10^{-2} \mathrm{~T}=79$ milligauss and these values indeed give $v_{\mathrm{en}} / \omega_{\mathrm{ce}}=1 / 4$. The ion-neutral collision frequency is

$v_{\text {in }}=n_{\mathrm{n}}\langle\sigma v\rangle_{\mathrm{in}}$,

where $\langle\sigma v\rangle_{\text {in }}=1.9 \times 10^{-15} \mathrm{~m}^{3} \mathrm{~s}^{-1}$ (Balbus \& Terquem 2001) and so for $n_{\mathrm{n}}=6 \times 10^{19} \mathrm{~m}^{-3}$, the ion-neutral collision frequency is $v_{\text {in }}=1.1 \times 10^{5} \mathrm{~s}^{-1}$. The ratio of ion-neutral collisions to ion cyclotron frequency can then be expressed as

$\frac{v_{\text {in }}}{\omega_{\mathrm{ci}}}=\frac{\omega_{\mathrm{ce}}}{4\langle\sigma v\rangle_{\mathrm{en}}} \frac{\langle\sigma v\rangle_{\mathrm{in}}}{\omega_{\mathrm{ci}}}=\frac{1}{4} \frac{m_{\mathrm{i}}}{m_{\mathrm{e}}} \frac{\langle\sigma v\rangle_{\mathrm{in}}}{\langle\sigma v\rangle_{\mathrm{en}}}=\frac{10^{3}}{T^{1 / 2}}$

so $v_{\text {in }} / \omega_{\text {ci }} \simeq 1.5 \times 10^{2} \gg 1$ for $T=48 \mathrm{~K}$. Thus, the system is indeed in the regime where $\omega_{\text {ci }} / v_{\text {in }} \ll 1 \ll \omega_{\text {ce }} / \nu_{\text {en }}$, i.e. the ions are collisionally bound to the neutrals while the electrons are frozen to the poloidal flux surfaces.

\section{TOROIDAL CURRENT}

We note from equation (36) that the poloidal magnetic field vanishes where $\nabla \psi=0$, i.e. at a maximum or minimum of $\psi$. The location of the black circle in Fig. 6 is where $\psi$ is at a minimum and so $\boldsymbol{B}_{\mathrm{pol}}=0$ at the black circle. Because $I(r, z, t)$ was assumed to be an odd function of $z$, it is seen that $B_{\phi}=0$ at all radii in the $z=0$ plane. In particular, $B_{\phi}$ vanishes at the black circle which thus is a magnetic field null. There are consequently no magnetic forces at the black circle so plasma at this location could be in a Kepler orbit. As shown by equation (47) a toroidal current must flow at the black circle to produce the poloidal magnetic field. This toroidal current results from a difference between the electron and ion toroidal velocities so the ions (and their associated collisionally coupled neutrals) could rotate at the Kepler velocity while associated neutralizing electrons rotate at a somewhat faster velocity to produce the required negative toroidal current.

\section{SUMMARY}

An axisymmetric topology has been presented consistent with accretion, jets, magnetic fields, electric circuit considerations, power sourcing/sinking, blackbody radiation from the disc and angular momentum transport. The integrated model shows how accretion drives astrophysical jets while overall angular momentum is conserved. Hall physics in the accretion disc provides an electric power source in the accretion disc while ideal MHD physics outside the accretion disc provides an electric power sink outside. The heterogeneity of the system (weakly ionized plasma in the vicinity of the $z=0$ plane, nearly fully ionized plasma away from $z=0$ plane) is crucial; the model would not work if the system were weakly ionized everywhere or were fully ionized everywhere. This heterogeneity (i.e. stratification of $\alpha$ ) is consistent with actual disc-jet systems. The $-r J_{r} B_{z}$ torque in the $z=0$ plane transfers angular momentum from small $r$ where $B_{z}$ is negative to large $r$ where $B_{z}$ is positive; the torque transfer is done electrically, not mechanically. It is like the countertorque acting on a hydroelectric generator at a dam absorbing the angular momentum of falling water and then transmitting this via cables to a distant motor that spins a propeller in a pond to create angular momentum in the pond. At the hydroelectric generator, it appears as if angular momentum is not being conserved and similarly it appears as if angular momentum is not being conserved at the pond, but when the electrical transmission is taken into account it is seen that angular momentum is conserved overall; the cables between generator and motor transport not only electric power but also angular momentum.

\section{ACKNOWLEDGEMENTS}

This material is based upon work supported by the U.S. Department of Energy Office of Science, Office of Fusion Energy Sciences under Award Numbers DE-FG02-04ER54755 and DE-SC0010471. The author wishes to thank an anonymous reviewer for pointing out that a range of particles with finite $P_{\phi}$ can have unstable circular orbits when $\beta=1$ as demonstrated in Appendix A.

\section{REFERENCES}

Aschwanden M. J., 1998, ApJ, 502, 455

Bai X. N., 2011, ApJ, 739, 51

Bai X. N., 2014a, ApJ, 791, 72

Bai X. N., 2014b, ApJ, 791, 137

Bai X. N., 2015, ApJ, 798, 84

Balbus S. A., 2003, ARA\&A, 41, 555

Balbus S. A., Terquem C., 2001, ApJ, 552, 235

Bellan P. M., 2000, Spheromaks: A Practical Application of Magnetohydrodynamic Dynamos and Plasma Self-organization. Imperial College Press, London

Bellan P. M., 2007, Phys. Plasmas, 14, 122901

Bellan P. M., 2008a, ApJ, 687, 311

Bellan P. M., 2008b, Fundamentals of Plasma Physics, paperback edition. Cambridge Univ. Press, Cambridge

Bellan P. M., 2016, J. Plasma Phys., 82, 615820101

Blandford R. D., Payne D. G., 1982, MNRAS, 199, 883

Chen F. F., 1984, Introduction to Plasma Physics and Controlled Fusion, 2nd edn. Plenum Press, New York 
Coffey D., Bacciotti F., Podio L., 2008, ApJ, 689, 1112

Freidberg J. P., 1987, Ideal Magnetohydrodynamics. Plenum Press, New York

Gammie C. F., 1996, ApJ, 457, 355

Goldstein H., 1950, Classical Mechanics. Addison Wesley, Reading, MA

Hartmann L., 2009, Accretion Process in Star Formation, 2nd edn. Cambridge Univ. Press, Cambridge

Hawley J. F., Gammie C. F., Balbus S. A., 1995, ApJ, 440, 742

Hsu S. C., Bellan P. M., 2002, MNRAS, 334, 257

Konigl A., 2010, MNRAS, 407, L79

Kulsrud R. M., 2005, Plasma Physics for Astrophysicists. Princeton Univ. Press, Princeton, NJ

Kumar D., Bellan P. M., 2009, Phys. Rev. Lett., 103, 105003

Kunz M. W., 2008, MNRAS, 385, 1494

Kunz M. W., Lesur G., 2013, MNRAS, 434, 2295

Landau L. D., Lifshits E. M., 1960, Mechanics. Pergamon Press, Oxford

Lesur G., Kunz M. W., Fromang S., 2014, A\&A, 566, A56

Lynden-Bell D., 2003, MNRAS, 341, 1360

Moser A. L., Bellan P. M., 2012, Nature, 482, 379

O'Keeffe W., Downes T. P., 2014, MNRAS, 441, 571

Pandey B. P., Wardle M., 2008, MNRAS, 385, 2269

Papaloizou J. C. B., Lin D. N. C., 1995, ARA\&A, 33, 505

Salmeron R., Wardle M., 2008, MNRAS, 388, 1223

Sano T., Stone J. M., 2002a, ApJ, 570, 314

Sano T., Stone J. M., 2002b, ApJ, 577, 534

Sano T., Miyama S. M., Umebayashi T., Nakano T., 2000, ApJ, 543, 486

Schmidt G., 1979, Physics of High Temperature Plasmas. Academic Press, New York, p. 29

Spitzer L., 1956, Physics of Fully Ionized Gases. Interscience, New York

Stone J. M., Hawley J. F., Gammie C. F., Balbus S. A., 1996, ApJ, 463, 656

Tsukamoto Y., Iwasaki K., Okuzumi S., Machida M. N., Inutsuka S., 2015, ApJ, 810, L26

Tsurutani B. T., Lakhina G. S., 1997, Rev. Geophys., 35, 491

Wardle M., 1999, MNRAS, 307, 849

You S., Yun G. S., Bellan P. M., 2005, Phys. Rev. Lett., 95, 045002

Zhai X., Li H., Bellan P. M., Li S. T., 2014, ApJ, 791, 40

\section{APPENDIX A: UNSTABLE CIRCULAR ORBITS WITH $\beta=1$ AND FINITE $P_{\phi}$}

Let assume $\beta=1$ so the effective potential, equation (26), becomes

$\chi(\bar{r})=\left(\frac{1}{2 \bar{r}^{2}}+2 \bar{r}^{1 / 2}\right) \bar{\omega}_{\mathrm{c}}+\frac{1}{2 \bar{r}^{2}}-\frac{1}{\bar{r}}+\left(\frac{1}{2 \bar{r}^{2}}+\frac{\bar{r}^{2}}{2}\right) \frac{\bar{\omega}_{\mathrm{c}}^{2}}{4}$.

The first and second derivatives of $\chi$ are

$\frac{\mathrm{d} \chi}{\mathrm{d} \bar{r}}=\left(-\frac{1}{\bar{r}^{3}}+\bar{r}^{-1 / 2}\right) \bar{\omega}_{\mathrm{c}}-\frac{1}{\bar{r}^{3}}+\frac{1}{\bar{r}^{2}}+\left(-\frac{1}{\bar{r}^{3}}+\bar{r}\right) \frac{\bar{\omega}_{\mathrm{c}}^{2}}{4}$

and

$\frac{\mathrm{d}^{2} \chi}{\mathrm{d} \bar{r}^{2}}=\left(\frac{3}{\bar{r}^{4}}-\frac{1}{2} \bar{r}^{-3 / 2}\right) \bar{\omega}_{\mathrm{c}}+\frac{3}{\bar{r}^{4}}-\frac{2}{\bar{r}^{3}}+\left(\frac{3}{\bar{r}^{4}}+1\right) \frac{\bar{\omega}_{\mathrm{c}}^{2}}{4}$.

Radial force balance and hence a circular orbit equilibrium corresponds to $\mathrm{d} \chi / \mathrm{d} \bar{r}=0$ at $\bar{r}=1$ which is satisfied by setting $\bar{r}=1$ in equation (A2). This circular orbit is a stable equilibrium if, in addition, the effective potential is at a local minimum (valley) at $\bar{r}=1$, i.e. if $\mathrm{d}^{2} \chi / \mathrm{d} \bar{r}^{2}>0$ at $\bar{r}=1$. Evaluating equation (A3) at $\bar{r}=1$ gives the condition for the circular orbit to be stable as

$\frac{\mathrm{d}^{2} \chi}{\mathrm{d} \bar{r}^{2}}=\frac{5}{2} \bar{\omega}_{\mathrm{c}}+1+\bar{\omega}_{\mathrm{c}}^{2}=\left(\bar{\omega}_{\mathrm{c}}+2\right)\left(\bar{\omega}_{\mathrm{c}}+1 / 2\right)>0$,

which indicates that unstable circular orbit equilibria occur if $-2 \leq \bar{\omega}_{\mathrm{c}} \leq-1 / 2$. Since a particle on a circular orbit starts with $\mathrm{d} \phi / \mathrm{d} \tau=1$ and $\bar{r}=1$, the normalized canonical angular momentum is

$\frac{P_{\phi}}{m \omega_{\mathrm{K} 0} r_{0}^{2}}=\frac{m r^{2} \dot{\phi}+q B_{z} r^{2} / 2}{m \omega_{\mathrm{K} 0} r_{0}^{2}}=\bar{r}^{2}\left(\frac{\mathrm{d} \phi}{\mathrm{d} \tau}+\frac{\bar{\omega}_{\mathrm{c}}}{2}\right)=1+\frac{\bar{\omega}_{\mathrm{c}}}{2}$.

Thus, $\quad P_{\phi} /\left(m \omega_{\mathrm{K} 0} r_{0}^{2}\right)=0$ occurs when $\bar{\omega}_{\mathrm{c}}=-2$ and $P_{\phi} /\left(m \omega_{\mathrm{K} 0} r_{0}^{2}\right)=3 / 4$ occurs when $\bar{\omega}_{\mathrm{c}}=-1 / 2$; equation (A5) shows that the range $-2 \leq \bar{\omega}_{\mathrm{c}} \leq-1 / 2$ maps to $0 \leq P_{\phi} \leq 3 m \omega_{\mathrm{K} 0} r_{0}^{2} / 4$.

A particle having $P_{\phi} /\left(m \omega_{\mathrm{K} 0} r_{0}^{2}\right)=3 / 4$ and associated $\bar{\omega}_{\mathrm{c}}=$ $-1 / 2$ in a field with $\beta=1$ will spiral towards larger radii when slightly perturbed. Such a particle spirals up to a radius $\bar{r}_{2}$ substantially larger than unity, then reflects and spirals down to a radius $\bar{r}_{1}$ that is infinitesimally less than unity where it reflects again. This can be seen by inserting $\bar{\omega}_{\mathrm{c}}=-1 / 2$ in $\chi(\bar{r})$ in equation (A1) to give the effective potential at $\bar{r}=1$ to be $\chi(1)=-27 / 16=-1.6875$. If the particle starting at $\bar{r}=1$ has zero initial radial velocity, it will stay at $\bar{r}=1$ forever and have a circular orbit. However, if it starts at $\bar{r}=1$ with an infinitesimal positive velocity it will spiral outwards until it reaches $\bar{r} \simeq 4.6$ where again $\chi=-1.6875$. At $\bar{r} \simeq 4.6$ the particle will reflect and move radially inwards until it reaches a point infinitesimally to the left of $\bar{r}=1$ where it will reflect again. Thus, a particle with $\bar{P}_{\phi}=3 / 4$ in a $\beta=1$ radial electric field moves on a circular orbit at $\bar{r}=1$ if it has zero initial radial velocity, but if it has infinitesimal initial radial velocity it will bounce back and forth radially between a point slightly to the left of $\bar{r}=1$ and $\bar{r} \simeq 4.6$.

\section{APPENDIX B: FLUID EQUIVALENT MODEL SHOWING CONSERVATION OF CANONICAL ANGULAR MOMENTUM}

We start from the Vlasov equation for each species and define species pressures in terms of random velocities relative to $\boldsymbol{U}$ the centre-of-mass velocity of the entire system. There are three species, electrons, ions and neutrals with each characterized by a velocity distribution function $f_{\sigma}(\boldsymbol{x}, \boldsymbol{v}, t)$. The density of each species is defined as

$n_{\sigma}(\boldsymbol{x}, t)=\int f_{\sigma}(\boldsymbol{x}, \boldsymbol{v}, t) \mathrm{d}^{3} v$

and the mass density of the complete fluid is defined as

$\rho(\boldsymbol{x}, t)=\sum_{\sigma} m_{\sigma} n_{\sigma}(\boldsymbol{x}, t)$

Because $m_{\mathrm{e}} \ll m_{\mathrm{i}}, m_{\mathrm{n}}$ it is seen that to a very high accuracy

$\rho(\boldsymbol{x}, t) \simeq m_{\mathrm{i}} n_{\mathrm{i}}+m_{\mathrm{n}} n_{\mathrm{n}}$.

The mean velocity of each species is defined as

$\boldsymbol{u}_{\sigma}(\boldsymbol{x}, t)=\frac{1}{n_{\sigma}(\boldsymbol{x}, t)} \int \boldsymbol{v} f_{\sigma}(\boldsymbol{x}, \boldsymbol{v}, t) \mathrm{d}^{3} v$

and because $m_{\mathrm{e}} \ll m_{\mathrm{i}}, m_{\mathrm{n}}$, the centre-of-mass velocity of the entire fluid to a very high accuracy is given by

$\boldsymbol{U}=\frac{m_{\mathrm{i}} n_{\mathrm{i}} \boldsymbol{u}_{\mathrm{i}}+m_{\mathrm{n}} n_{\mathrm{n}} \boldsymbol{u}_{\mathrm{n}}}{\rho(\boldsymbol{x}, t)}$.

Each species is assumed to have an isotropic pressure defined as

$P_{\sigma}=\frac{1}{3} \int m_{\sigma}\left|\boldsymbol{v}^{\prime}\right|^{2} f_{\sigma} \mathrm{d}^{3} v$,

where

$\boldsymbol{v}^{\prime}=\boldsymbol{v}-\boldsymbol{U}$ 
is the random velocity with respect to the centre of mass of the entire fluid. It is critical to note that $P_{\sigma}$ is defined in terms of a random velocity relative to the centre-of-mass velocity $\boldsymbol{U}$ of the entire fluid, not relative to $\boldsymbol{u}_{\sigma}$ the centre-of-mass velocity of species $\sigma$.

The pressure of the complete fluid is defined as

$P=\sum_{\sigma=\mathrm{e}, \mathrm{i}, \mathrm{n}} P_{\sigma}$

The Vlasov equation for each species is

$$
\begin{aligned}
\frac{\partial f_{\sigma}}{\partial t} & +\nabla \cdot\left(\boldsymbol{v} f_{\sigma}\right) \\
& +\frac{\partial}{\partial \boldsymbol{v}} \cdot\left[\left(q_{\sigma} \boldsymbol{E}+q_{\sigma} \boldsymbol{v} \times \boldsymbol{B}+m_{\sigma} \boldsymbol{g}\right) f_{\sigma}\right]=\sum_{\alpha \neq \sigma} C_{\sigma \alpha},
\end{aligned}
$$

where $\boldsymbol{g}$ is gravitational acceleration and $C_{\sigma \alpha}$ characterizes the change in $f_{\sigma}$ resulting from collisions of particles of species $\sigma$ with particles of species $\alpha$. Collisions do not change the number of particles so

$$
\int C_{\sigma \alpha} \mathrm{d}^{3} v=0
$$

When particles of species $\sigma$ collide with particles of species $\alpha$ the momentum lost by the $\sigma$ particles is gained by the $\alpha$ particles so

$\int m_{\sigma} v C_{\sigma \alpha} \mathrm{d}^{3} v+\int m_{\alpha} v C_{\alpha \sigma} \mathrm{d}^{3} v=0$.

If the collisions of $\sigma$ particles with $\alpha$ particles are represented by a nominal average collision frequency $v_{\sigma \alpha}$, then

$$
\int m_{\sigma} \boldsymbol{v} C_{\sigma \alpha} \mathrm{d}^{3} v=-m_{\sigma} n_{\sigma} v_{\sigma \alpha}\left(\boldsymbol{u}_{\sigma}-\boldsymbol{u}_{\alpha}\right)
$$

which shows that the $\sigma$ particles experience a drag if the $\sigma$ particles are going faster than the $\alpha$ particles. Equation (B12) can be considered to be the rate of change of momentum of species $\sigma$ as measured by an observer who is in a frame moving with the centre of mass of the $\alpha$ particles; this rate of change is the collision frequency $v_{\sigma \alpha}$ and it is important to realize that its definition requires specification of the $\alpha$ frame. Equations (B11) and (B12) then show that

$m_{\sigma} n_{\sigma} v_{\sigma \alpha}\left(\boldsymbol{u}_{\sigma}-\boldsymbol{u}_{\alpha}\right)+m_{\alpha} n_{\alpha} v_{\alpha \sigma}\left(\boldsymbol{u}_{\alpha}-\boldsymbol{u}_{\sigma}\right)=0$

SO

$m_{\sigma} n_{\sigma} v_{\sigma \alpha}=m_{\alpha} n_{\alpha} v_{\alpha \sigma}$.

In an electron-ion plasma where $n_{\mathrm{e}}=n_{\mathrm{i}}$, this reduces to the well-known result that $m_{\mathrm{e}} v_{\mathrm{ei}}=m_{\mathrm{i}} v_{\mathrm{ie}}$. The zeroth moment of equation (B9) is obtained by simply integrating over velocity. The term involving $\partial / \partial v$ becomes a surface integral at infinity in velocity space and vanishes because $f_{\sigma}$ vanishes for infinite velocity. The application of equations (B1), (B4) and (B10) gives the continuity equations for each species, namely

$\frac{\partial n_{\sigma}}{\partial t}+\nabla \cdot\left(n_{\sigma} \boldsymbol{u}_{\sigma}\right)=0$.

Multiplying by $m_{\sigma}$ and summing over all species gives the MHD continuity equation

$\frac{\partial \rho}{\partial t}+\nabla \cdot(\rho \boldsymbol{U})=0$
Next we multiply equation (B9) by $m_{\sigma} \boldsymbol{v}$ and integrate over velocity to obtain

$$
\left(\begin{array}{c}
\frac{\partial}{\partial t} \int m_{\sigma} \boldsymbol{v} f_{\sigma}(\boldsymbol{x}, \boldsymbol{v}, t) \mathrm{d}^{3} v \\
+\nabla \cdot\left(\int m_{\sigma} \boldsymbol{v} \boldsymbol{v} f_{\sigma}(\boldsymbol{x}, \boldsymbol{v}, t) \mathrm{d}^{3} v\right) \\
+\int \boldsymbol{v} \frac{\partial}{\partial \boldsymbol{v}} \cdot\left[\left(\begin{array}{c}
q_{\sigma} \boldsymbol{E} \\
+q_{\sigma} \boldsymbol{v} \times \boldsymbol{B} \\
+m \boldsymbol{g}
\end{array}\right) f_{\sigma}\right] \mathrm{d}^{3} v
\end{array}\right)=\sum_{\alpha \neq \sigma} \int m_{\sigma} \boldsymbol{v} C_{\sigma \alpha} \mathrm{d}^{3} v
$$

The integral in the second term of the left-hand side of equation (B17) is evaluated as

$$
\begin{aligned}
\int m_{\sigma} \boldsymbol{v} \boldsymbol{v} f_{\sigma}(\boldsymbol{x}, \boldsymbol{v}, t) \mathrm{d}^{3} v & =\int m_{\sigma}\left(\boldsymbol{v}^{\prime}+\boldsymbol{U}\right)\left(\boldsymbol{v}^{\prime}+\boldsymbol{U}\right) f_{\sigma} \mathrm{d}^{3} v \\
& =\int m_{\sigma}\left(\begin{array}{c}
\boldsymbol{v}^{\prime} \boldsymbol{v}^{\prime}+\boldsymbol{U} \boldsymbol{v}^{\prime} \\
+\boldsymbol{v}^{\prime} \boldsymbol{U}+\boldsymbol{U} \boldsymbol{U}
\end{array}\right) f_{\sigma} \mathrm{d}^{3} v \\
& =\left(\begin{array}{c}
P_{\sigma} \boldsymbol{I} \\
+\boldsymbol{U} \int m_{\sigma} \boldsymbol{v}^{\prime} f_{\sigma} \mathrm{d}^{3} v \\
+\int m_{\sigma} \boldsymbol{v}^{\prime} f_{\sigma} \mathrm{d}^{3} v \boldsymbol{U} \\
+m_{\sigma} n_{\sigma} \boldsymbol{U}
\end{array}\right) \boldsymbol{U} .
\end{aligned}
$$

We note that because the random velocities were defined with reference to $\boldsymbol{U}$

$$
\begin{aligned}
\sum_{\sigma} \int m_{\sigma} \boldsymbol{v}^{\prime} f_{\sigma} \mathrm{d}^{3} v & =\sum_{\sigma} \int m_{\sigma}(\boldsymbol{v}-\boldsymbol{U}) f_{\sigma} \mathrm{d}^{3} v \\
& =\sum_{\sigma}\left(m_{\sigma} n_{\sigma} \boldsymbol{u}_{\sigma}-m_{\sigma} n_{\sigma} \boldsymbol{U}\right) \\
& =\left(\sum_{\sigma} m_{\sigma} n_{\sigma} \boldsymbol{u}_{\sigma}\right)-\rho \boldsymbol{U} \\
& =0 .
\end{aligned}
$$

The third term on the left-hand side of equation (B17) can be integrated by parts in velocity space to give

$$
\begin{aligned}
\int m_{\sigma} \boldsymbol{v} \frac{\partial}{\partial \boldsymbol{v}} & \cdot\left[\left(\begin{array}{c}
q_{\sigma} \boldsymbol{E}+ \\
q_{\sigma} \boldsymbol{v} \times \boldsymbol{B} \\
+m_{\sigma} \boldsymbol{g}
\end{array}\right) f_{\sigma}\right] \mathrm{d}^{3} v \\
= & -\int\left(\begin{array}{c}
q_{\sigma} \boldsymbol{E} \\
+q_{\sigma} \boldsymbol{v} \times \boldsymbol{B} \\
+m_{\sigma} \boldsymbol{g}
\end{array}\right) f_{\sigma} \mathrm{d}^{3} v \\
= & \left(\begin{array}{c}
-n_{\sigma} q_{\sigma} \boldsymbol{E} \\
-n_{\sigma} q_{\sigma} \boldsymbol{u}_{\sigma} \times \boldsymbol{B} \\
-n_{\sigma} m_{\sigma} \boldsymbol{g}
\end{array}\right) .
\end{aligned}
$$

Summing equation (B17) over all species, defining $\boldsymbol{J}=$ $\sum n_{\sigma} q_{\sigma} \boldsymbol{u}_{\sigma}$, and assuming quasi-neutrality gives the MHD equation of motion

$\frac{\partial}{\partial t}(\rho \boldsymbol{U})+\nabla \cdot(\rho \boldsymbol{U} \boldsymbol{U})=\boldsymbol{J} \times \boldsymbol{B}-\nabla P+\rho \boldsymbol{g}$ 
or after subtracting $\boldsymbol{U}$ times equation (B16) from the left-hand side,

$\rho\left(\frac{\partial \boldsymbol{U}}{\partial t}+\boldsymbol{U} \cdot \nabla \boldsymbol{U}\right)=\boldsymbol{J} \times \boldsymbol{B}-\nabla P+\rho \boldsymbol{g}$.

The collision terms on the right-hand side cancel each other on equation (B17) over all species because the momentum lost by one species in a collision is gained by another and the sum is over all species.

Let us now make the following slight change in the procedure. We ignore the electron mass in the first term of equation (B17) and in the gravitational term but retain it in the other terms. This corresponds to assuming that $m_{\mathrm{e}} n_{\mathrm{e}} \boldsymbol{u}_{\mathrm{e}}$ is negligible compared to $m_{\mathrm{i}} n_{\mathrm{i}} \boldsymbol{u}_{\mathrm{i}}, m_{\mathrm{n}} n_{\mathrm{n}} \boldsymbol{u}_{\mathrm{n}}$ and assuming that $m_{\mathrm{e}} n_{\mathrm{e}}$ is negligible compared to $m_{\mathrm{i}} n, m_{\mathrm{n}} n_{\mathrm{n}}$. We also ignore the term containing electron mass in equation (B19) which is acceptable if $P_{\mathrm{e}}$ is of the order of $P_{\mathrm{i}}$ and $P_{\mathrm{n}}$ since if $P_{\mathrm{e}}=P_{\mathrm{i}}$, then $\left(m_{\mathrm{e}} v_{\mathrm{e}}^{\prime}\right)^{2}=\left(m_{\mathrm{e}} / m_{\mathrm{i}}\right)\left(m_{\mathrm{i}} v_{\mathrm{i}}^{\prime}\right)^{2}$ and so $m_{\mathrm{e}} v_{\mathrm{e}}^{\prime} \ll m_{\mathrm{i}} v_{\mathrm{i}}^{\prime}$.

Furthermore, instead of summing equation (B17) over all species, let us now (i) sum equation (B17) over ions and neutrals, and (ii) separately consider the electron equation resulting from equation (B17). In this case, summing over ions and neutrals will give the same left-hand side as equation (B22), but the acceleration term given by equation (B20) will be retained as will the collision terms with electrons.

Thus, summing equation (B17) over just ions and neutrals gives

$\rho\left(\frac{\partial \boldsymbol{U}}{\partial t}+\boldsymbol{U} \cdot \nabla \boldsymbol{U}\right)=\left(\begin{array}{c}n_{\mathrm{i}} q_{\mathrm{i}} \boldsymbol{E}+n_{\mathrm{i}} q_{\mathrm{i}} \boldsymbol{u}_{\mathrm{i}} \times \boldsymbol{B} \\ +\rho \boldsymbol{g}-m_{\mathrm{i}} n_{\mathrm{i}} v_{i e}\left(\boldsymbol{u}_{\mathrm{i}}-\boldsymbol{u}_{\mathrm{e}}\right) \\ -m_{\mathrm{n}} n_{\mathrm{n}} v_{n e}\left(\boldsymbol{u}_{\mathrm{n}}-\boldsymbol{u}_{\mathrm{e}}\right) \\ -\nabla\left(P_{\mathrm{i}}+P_{\mathrm{n}}\right)\end{array}\right)$

while the separate electron equation is

$0=\left(\begin{array}{c}n_{\mathrm{e}} q_{\mathrm{e}} \boldsymbol{E}+n_{\mathrm{e}} q_{\mathrm{e}} \boldsymbol{u}_{\mathrm{e}} \times \boldsymbol{B} \\ -m_{\mathrm{e}} n_{\mathrm{e}} v_{e i}\left(\boldsymbol{u}_{\mathrm{e}}-\boldsymbol{u}_{\mathrm{i}}\right)-m_{\mathrm{e}} n_{\mathrm{e}} v_{\mathrm{en}}\left(\boldsymbol{u}_{\mathrm{e}}-\boldsymbol{u}_{\mathrm{n}}\right) \\ -\nabla P_{\mathrm{e}}\end{array}\right)$.

Because the ions have nearly the same mass as the neutrals, ions efficiently exchange momentum with neutrals and with each other. It is assumed that the collision rate of ions with neutrals greatly exceeds the ion cyclotron frequency so the ion adiabatic invariant $\mu=m v_{\perp}^{2} /(2 B)$ is not conserved, individual ions are not flux conservers and ions are not constrained to stay on magnetic flux surfaces (Pandey \& Wardle 2008). Since the gas is weakly ionized, an ion collides much more frequently with neutrals than with other ions or with electrons. Any momentum an ion gains from electromagnetic forces is quickly shared with neutrals but not with electrons (Pandey \& Wardle 2008).

This high rate of exchange of momentum between ions and neutrals means that $\boldsymbol{u}_{\mathrm{i}} \simeq \boldsymbol{u}_{\mathrm{n}}$ and so

$\boldsymbol{u}_{\mathrm{e}}-\boldsymbol{u}_{\mathrm{n}} \simeq \boldsymbol{u}_{\mathrm{e}}-\boldsymbol{u}_{\mathrm{i}}$

However, the current density is given by

$\boldsymbol{J}=n_{\mathrm{i}} q_{\mathrm{i}} \boldsymbol{u}_{\mathrm{i}}+n_{\mathrm{e}} q_{\mathrm{e}} \boldsymbol{u}_{\mathrm{e}}$

and because of the very low fractional ionization and the assumed quasi-neutrality all ions are singly ionized, so

$\boldsymbol{J}=n_{\mathrm{i}} e\left(\boldsymbol{u}_{\mathrm{i}}-\boldsymbol{u}_{\mathrm{e}}\right)$

The electron drag term can be expressed as

$$
\begin{aligned}
\left(\begin{array}{c}
m_{\mathrm{e}} n_{\mathrm{e}} v_{\mathrm{ei}}\left(\boldsymbol{u}_{\mathrm{e}}-\boldsymbol{u}_{\mathrm{i}}\right) \\
+m_{\mathrm{e}} n_{\mathrm{e}} v_{\mathrm{en}}\left(\boldsymbol{u}_{\mathrm{e}}-\boldsymbol{u}_{\mathrm{n}}\right)
\end{array}\right) & =m_{\mathrm{e}} n_{\mathrm{e}}\left(v_{\mathrm{ei}}+v_{\mathrm{en}}\right)\left(\boldsymbol{u}_{\mathrm{e}}-\boldsymbol{u}_{\mathrm{i}}\right) \\
& =-n_{\mathrm{e}} e \eta \boldsymbol{J},
\end{aligned}
$$

where

$\eta=\frac{m_{\mathrm{e}}\left(v_{\mathrm{ei}}+v_{\mathrm{en}}\right)}{n_{\mathrm{e}} e^{2}}$

is the electrical resistivity. Equations (B23) and (B24) can then be written as

$\rho\left(\frac{\partial \boldsymbol{U}}{\partial t}+\boldsymbol{U} \cdot \nabla \boldsymbol{U}\right)=\left(\begin{array}{c}n_{\mathrm{i}} e \boldsymbol{E}+n_{\mathrm{i}} e \boldsymbol{u}_{\mathrm{i}} \times \boldsymbol{B} \\ +\rho \boldsymbol{g}-n_{\mathrm{e}} e \eta \boldsymbol{J} \\ -\nabla\left(P_{\mathrm{i}}+P_{\mathrm{n}}\right)\end{array}\right)$

$0=\left(\begin{array}{c}-n_{\mathrm{e}} e \boldsymbol{E}-n_{\mathrm{e}} e \boldsymbol{u}_{\mathrm{e}} \times \boldsymbol{B} \\ +n_{\mathrm{e}} e \eta \boldsymbol{J}-\nabla P_{\mathrm{e}}\end{array}\right)$,

i.e. equations (61) and (62).

This paper has been typeset from a $\mathrm{T}_{\mathrm{E}} \mathrm{X} / \mathrm{LT} \mathrm{T} \mathrm{X}$ file prepared by the author. 